\title{
Reconstruction of 2-Convex Polyominoes with Non-Empty Corners
}

\author{
Khalil Tawbe ${ }^{1,2}$, Salwa Mansour ${ }^{3}$ \\ ${ }^{1}$ Department of Mathematics, Lebanese University, Beirut, Lebanon \\ ${ }^{2}$ Department of Mathematics, The International University of Beirut, Beirut, Lebanon \\ ${ }^{3}$ Department of Mathematics, Lebanese International University, Beirut, Lebanon \\ Email: Khaliltawbe@hotmail.com, salwa.mansour@liu.edu.lb
}

How to cite this paper: Tawbe, $K$. and Mansour, S. (2019) Reconstruction of 2-Convex Polyominoes with Non-Empty Corners. Open Journal of Discrete Mathematics, 9, 83-109.

https://doi.org/10.4236/ojdm.2019.94009

Received: May 5, 2019

Accepted: August 6, 2019

Published: August 9, 2019

Copyright (c) 2019 by author(s) and Scientific Research Publishing Inc. This work is licensed under the Creative Commons Attribution International License (CC BY 4.0).

http://creativecommons.org/licenses/by/4.0/

\begin{abstract}
This paper uses the theoretical material developed in a previous study by the authors in order to reconstruct a subclass of 2-convex polyominoes called $\alpha_{2 L}^{1,1}$ where the upper left corner and the lower right corner of the polyomino contain each only one cell. The main idea is to control the shape of these polyominoes by using 32 types of geometries. Some modifications are made in the reconstruction algorithm of Chrobak and Dürr for $H V$-convex polyominoes in order to impose these geometries.
\end{abstract}

\section{Keywords}

Polyomino, Convex Objects, Monotone Path

\section{Introduction}

The present paper uses the theoretical material developed in a previous study by the authors in order to reconstruct a subclass of 2-convex polyominoes. Indeed, 2 -convex polyominoes are the first difficult class of polyominoes in terms of tomographical reconstruction in the hierarchy of $k$-polyominoes and in this article we design an algorithm of reconstruction for a subclass of $L$-convex which is the second step in the whole comprehension of the hierarchy of $k$-polyominoes.

One main problem in discrete tomography consists on the reconstruction of discrete objects according to their horizontal and vertical projection vectors. In order to restrain the number of solutions, we could add convexity constraints to these discrete objects. There are many notions of discrete convexity of polyominoes (namely $H V$-convex [1], $Q$-convex [2], $L$-convex polyominoes [3]) and each one leads to interesting studies. One natural notion of convexity on the discrete plane is the class of $H V$-convex polyominoes that are polyominoes with 
consecutive cells in rows and columns. Following the work of Del Lungo, Nivat, Barcucci, and Pinzani [1] we are able using discrete tomography to reconstruct polyominoes that are $H V$-convex according to their horizontal and vertical projections. In addition to that, for an $H V$-convex polyomino $P$ every pairs of cells of $P$ can be reached using a path included in $P$ with only two kinds of unit steps (such a path is called monotone). A polyomino is called $k$-convex if for every two cells we find a monotone path with at most $k$ changes of direction. Obviously a $k$-convex polyomino is an $H V$-convex polyomino. Thus, the set of $k$-convex polyominoes for $k \in \mathbb{N}$ forms a hierarchy of $H V$-convex polyominoes according to the number of changes of direction of monotone paths. This notion of 1-convex polyominoes has been considered by several points of view. In [4] combinatorial aspects of 1-convex polyominoes are analyzed, giving the enumeration according to the semi-perimeter and the area. In [5] it is given an algorithm that reconstructs a 1-convex polyomino from the set of its maximal $L$-polyominoes. Similarly in [3] it is given another way to reconstruct a $L$-convex polyomino from the size of some special paths, called bordered $L$-paths.

In fact 2-convex polymoninoes are more geometrically complex and there was no result for their direct reconstruction. We could notice that Duchi, Rinaldi, and Schaeffer are able to enumerate this class in an interesting and technical article [6]. But the enumeration technique gives no idea for the tomographical reconstruction.

The first subclass that creates the link with 2-convex polyominoes is the class of $H V$-centered polyominoes. In [7], it is showed that if $P$ is an $H V$-centered polyomino then $P$ is 2-convex. Note that the tomographical properties of this subclass have been studied in [8] and its reconstruction algorithm is well known.

The main contribution of this paper is an $O\left(\mathrm{~m}^{3} \mathrm{n}^{3}\right)$-time algorithm for reconstructing a subclass of 2-convex polyominoes using the geometrical properties studied in a previous study, and the algorithm of Chrobak and Dürr [8]. In particular, we add well-chosen clauses to the original construction of Chrobak and Dürr in order to control the $2 L$-convexity using 2SAT satisfaction problem.

This paper is divided into 5 sections. After basics on polyominoes, Section 3 shows the different geometrical shapes of a subclass of 2-convex polyominoes [9]. In Section 4, the algorithm of Chrobak and Dür for the reconstruction of the $H V$-convex polyominoes is given [8]. Section 5 describes the reconstruction of different subclasses of 2-convex polyominoes.

\section{Definition and Notation}

A planar discrete set is a finite subset of the integer lattice $\mathbb{Z}^{2}$ defined up to translation. A discrete set can be represented either by a set of cells, i.e. unitary squares of the cartesian plane, or by a binary matrix, where the 1's determine the cells of the set (see Figure 1).

A polyomino $P$ is a finite connected set of adjacent cells, defined up to translations, in the cartesian plane. A row convex polyomino (resp. column-convex) is 


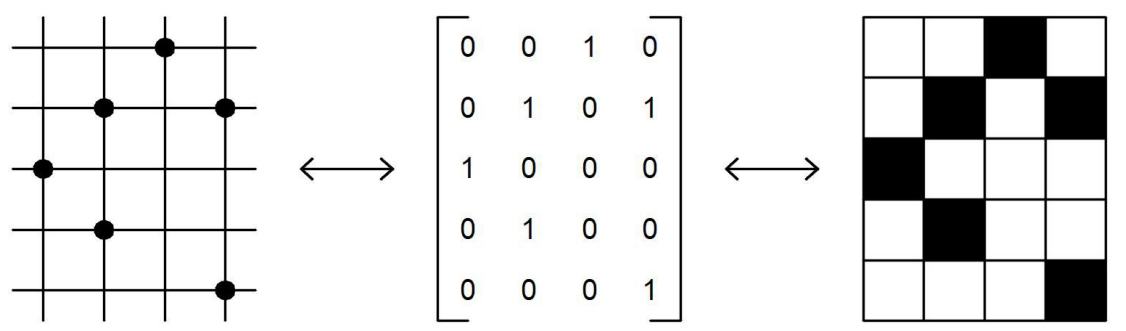

Figure 1. A finite set of $\mathbb{N} \times \mathbb{N}$, and its representation in terms of a binary matrix and a set of cells.

a self avoiding convex polyomino such that the intersection of any horizontal line (resp. vertical line) with the polyomino has at most two connected components. Finally, a polyomino is said to be convex (or $H V$-convex) if it is both row and column-convex (see Figure 2).

To each discrete set $S$, represented as a $m \times n$ binary matrix, two integer vectors $H=\left(h_{1}, \cdots, h_{m}\right)$ and $V=\left(v_{1}, \cdots, v_{n}\right)$ are associated such that, for each $1 \leq i \leq m, 1 \leq j \leq n, h_{i}$ and $v_{j}$ are the number of cells of $S$ (elements 1 of the matrix) which lie on row $i$ and column $j$, respectively. The vectors $H$ and $V$ are called the horizontal and vertical projections of $\mathcal{S}$, respectively (see Figure 3 ). Moreover if $S$ has $H$ and $V$ as horizontal and vertival projections, respectively, then we say that $S$ satisfies $(H, V)$. Using the usual matrix notations, the element $(i, j)$ denotes the entry in row $i$ and column $j$.

For any two cells $A$ and $B$ in a polyomino, a path $\Pi_{A B}$, from $A$ to $B$, is a sequence $\left(i_{1}, j_{1}\right),\left(i_{2}, j_{2}\right), \cdots,\left(i_{r}, j_{r}\right)$ of adjacent disjoint cells belonging in $P$, with $A=\left(i_{1}, j_{1}\right)$, and $B=\left(i_{r}, j_{r}\right)$. For each $1 \leq k \leq r-1$, we say that the two consecutive cells $\left(i_{k}, j_{k}\right),\left(i_{k+1}, j_{k+1}\right)$ form:

- an east step if $i_{k+1}=i_{k}$ and $j_{k+1}=j_{k}+1$;

- a north step if $i_{k+1}=i_{k}-1$ and $j_{k+1}=j_{k}$;

- a west step if $i_{k+1}=i_{k}$ and $j_{k+1}=j_{k}-1$;

- a south step if $i_{k+1}=i_{k}+1$ and $j_{k+1}=j_{k}$.

Finally, we define a path to be monotone if it is entirely made of only two of the four types of steps defined above (see [7] [10]).

Proposition 1 (Castiglione, Restivo). A polyomino $P$ is $H V$-convex if and only if every pair of cells is connected by a monotone path.

Let us consider a polyomino $P$. A path in $P$ has a change of direction in the cell $\left(i_{k}, j_{k}\right)$, for $2 \leq k \leq r-1$, if

$$
i_{k} \neq i_{k-1} \Leftrightarrow j_{k+1} \neq j_{k} .
$$

Definition 1. We call $k$-convex an $H V$-convex polyomino such that every pair of its cells can be connected by a monotone path with at most $k$ changes of direction respectively.

In [5], it is proposed a hierarchy on convex polyominoes based on the number of changes of direction in the paths connecting any two cells of a polyomino.

For $k=1$, we have the first level of hierarchy, i.e. the class of 1-convex polyominoes, also denoted $L$-convex polyominoes for the typical shape of each 

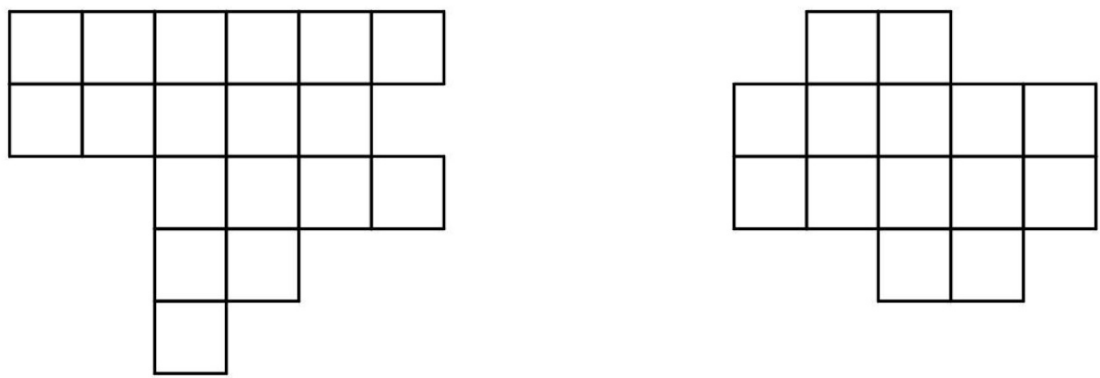

Figure 2. Row convex and convex polyomino.

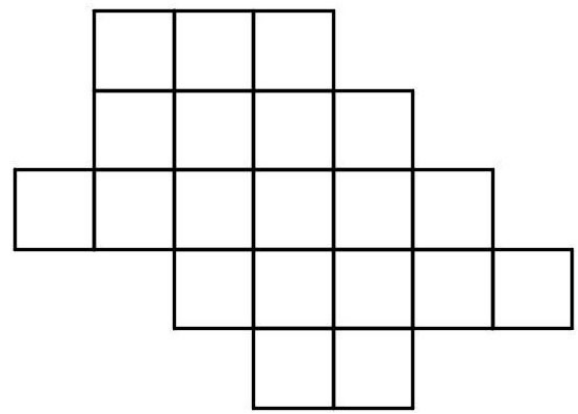

Figure 3. A polyomino $P$ with $H=(3,4,6,5,2)$ and $V=(1,3,4,5,4,1)$.

path having at most one single change of direction. In the present study, we focus our attention on the next level of the hierarchy, i.e. the class of 2-convex polyominoes, whose geometrical properties are more complicated and harder to be investigated than those of 1-convex polyominoes (see Figure 4).

\section{2-Convex Polyominoes}

Let $(H, V)$ be two projection vectors and let $P$ be an $H V$-convex polyomino, that satisfies $(H, V)$. By a classical argument $P$ is contained in a rectangle $R$ (called minimal bounding box) where in this box no projection gives a zero. Let $[\min (S), \max (S)]([\min (E), \max (E)],[\min (N), \max (N)]$, $[\min (W), \max (W)]$ ) be the intersection of $P$ s boundary on the lower (right, upper, left) side of $R$ (see [1]). By abuse of notation, we call $\min (S)$ [resp. $\min (E), \min (N), \min (W)]$ the cell at the position $(m, \min (S))$ [resp. $(\min (E), n),(1, \min (N)),(\min (W), 1)]$ and $\max (S)$ [resp. $\max (E)$, $\max (N), \max (W)]$ the cell at the position $(m, \max (S))$ [resp. $(\max (E), n)$, $(1, \max (N)),(\max (W), 1)]$ (see Figure 5$)$.

Definition 2. The segment $[\min (S), \max (S)]$ is called the $S$-foot. Similarly, the segments $[\min (E), \max (E)],[\min (N), \max (N)]$ and $[\min (W), \max (W)]$ are called $E$-foot, $N$-foot and $W$-foot.

For a bounding rectangle $R$ and for a given polyomino $P$, let us define the following sets:

- $W N=\{(i, j) \in P \mid i<\min (W)$ and $j<\min (N)\}$,

- $S E=\{(i, j) \in P \mid i>\max (E)$ and $j>\max (S)\}$, 

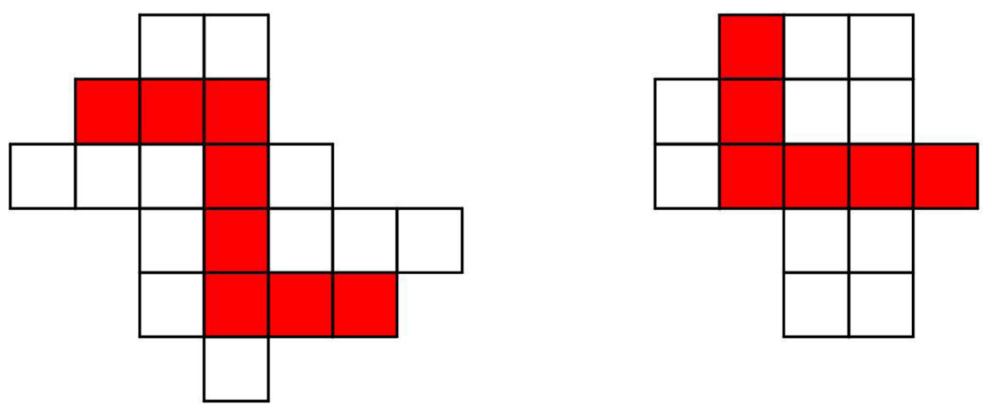

Figure 4. The convex polyomino on the left is 2-convex, while the one on the right is 1-convex.

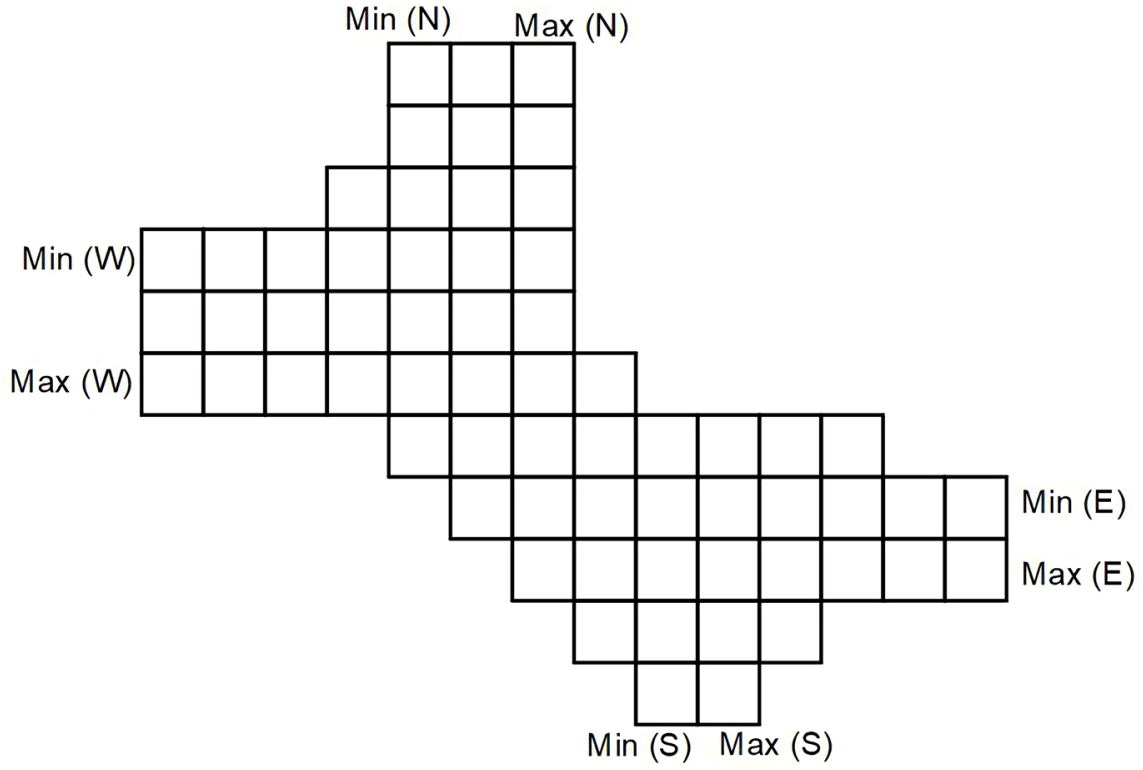

Figure 5. Min and max of the four feet.

- $N E=\{(i, j) \in P \mid i<\min (E)$ and $j>\max (N)\}$,

- $W S=\{(i, j) \in P \mid i>\max (W)$ and $j<\min (S)\}$.

Let $\mathcal{C}$ be the class of $H V$-convex polyominoes, thus we have the following classes of polyominoes regarding the position of the non-intersecting feet.

$$
\begin{aligned}
\alpha^{1,1}=\{ & P \in \mathcal{C} \mid \max (N)<\min (S) \text { and } \max (W)<\min (E) ; \\
& \operatorname{card}(W N)=1 \text { and } \operatorname{card}(S E)=1\} \\
\beta^{1,1}=\{ & P \in \mathcal{C} \mid \max (S)<\min (N) \text { and } \max (E)<\min (W) ; \\
& \operatorname{card}(N E)=1 \text { and } \operatorname{card}(W S)=1\} \\
\alpha_{2 L}^{1,1}= & \{P \in \mathcal{C} \mid \max (N)<\min ((S) \text { and } \max (W)<\min (E) ;, \text { where } P \text { is a } \\
& \operatorname{card}(W N)=1 \text { and } \operatorname{card}(S E)=1\}
\end{aligned}
$$

2-convex polyomino.

$\beta_{2 L}^{1,1}=\{P \in \mathcal{C} \mid \max (S)<\min (N)$ and $\max (E)<\min (W)$;

$$
\operatorname{card}(N E)=1 \text { and } \operatorname{card}(W S)=1\}
$$

2-convex polyomino. 
Now in order to understand the different geometries of the class $\alpha_{2 L}^{1,1}$, let us define the following subclasses of the class $\alpha^{1,1}$.

$\gamma^{1,1}=\{P \in \mathcal{C} \mid \max (N)<\min (S)$ and $\max (W)<\min (E) ; \operatorname{card}(W N)=1$

and $\operatorname{card}(S E)=1 ; \min (E)=\max (W)+1$ and $\min (S)=\max (N)+1\}$

Figure 6).

$\delta^{1,1}=\{P \in \mathcal{C} \mid \max (S)<\min (N)$ and $\max (E)<\min (W) ; \operatorname{card}(N E)=1$

and $\operatorname{card}(W S)=1 ; \min (E) \neq \max (W)+1$ and $\min (S)=\max (N)+1\} \quad$. (see

Figure 6).

$\Delta^{1,1}=\{P \in \mathcal{C} \mid \max (N)<\min (S)$ and $\max (W)<\min (E) ; \operatorname{card}(W N)=1$

- $\quad$ and $\operatorname{card}(S E)=1 ; \min (E)=\max (W)+1$ and $\min (S) \neq \max (N)+1\}$ (see

Figure 6).

$\chi^{1,1}=\{P \in \mathcal{C} \mid \max (N)<\min (S)$ and $\max (W)<\min (E) ; \operatorname{card}(W N)=1$

and $\operatorname{card}(S E)=1 ; \min (E) \neq \max (W)+1$ and $\min (S) \neq \max (N)+1\}$

Figure 6).

$\gamma_{2 L}^{1,1}=\{P \in \mathcal{C} \mid \max (N)<\min (S)$ and $\max (W)<\min (E) ; \operatorname{card}(W N)=1$

and $\operatorname{card}(S E)=1 ; \min (E)=\max (W)+1$ and $\min (S)=\max (N)+1\}$

where $P$ is a 2-convex polyomino.

$\delta_{2 L}^{1,1}=\{P \in \mathcal{C} \mid \max (S)<\min (N)$ and $\max (E)<\min (W) ; \operatorname{card}(N E)=1$

and $\operatorname{card}(W S)=1 ; \min (E) \neq \max (W)+1$ and $\min (S)=\max (N)+1\}$

where $P$ is a 2-convex polyomino.

$\Delta_{2 L}^{1,1}=\{P \in \mathcal{C} \mid \max (N)<\min (S)$ and $\max (W)<\min (E) ; \operatorname{card}(W N)=1$

and $\operatorname{card}(S E)=1 ; \min (E)=\max (W)+1$ and $\min (S) \neq \max (N)+1\}$

where $P$ is a 2 -convex polyomino.

$\chi_{2 L}^{1,1}=\{P \in \mathcal{C} \mid \max (N)<\min (S)$ and $\max (W)<\min (E) ; \operatorname{card}(W N)=1$

and $\operatorname{card}(S E)=1 ; \min (E) \neq \max (W)+1$ and $\min (S) \neq \max (N)+1\}$

where $P$ is a 2 -convex polyomino.

Theorem 1. Let $P$ be a convex polyomino in the class $\gamma^{1,1}, P$ is 2 -convex if and only if there exists an $L$-path from

1)

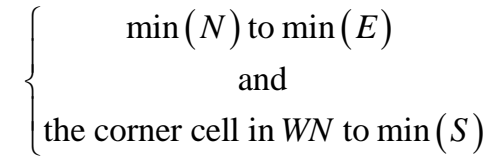

or

2)

$$
\left\{\begin{array}{c}
\min (W) \text { to } \min (S) \\
\text { and } \\
\text { the corner cell in } W N \text { to } \min (E)
\end{array}\right.
$$

or

3)

$$
\left\{\begin{array}{c}
\max (N) \text { to } \max (E) \\
\text { and } \\
\max (W) \text { to the corner cell in } S E
\end{array}\right.
$$


a)

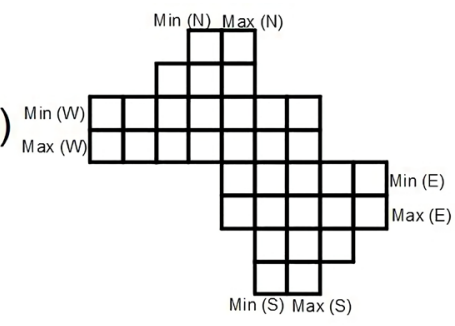

c)

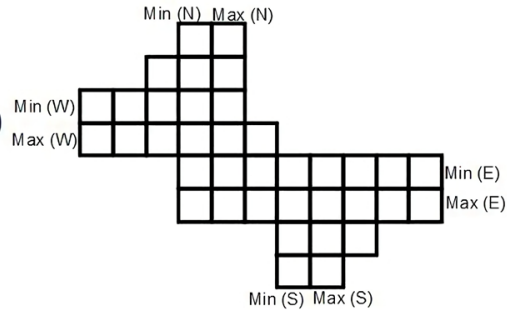

b)

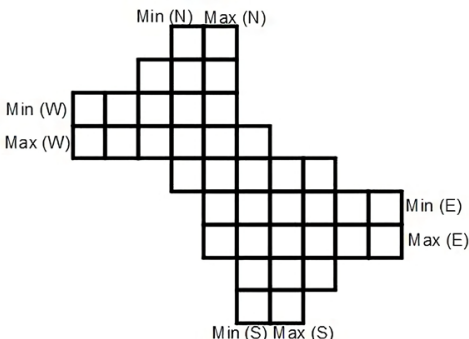

d)

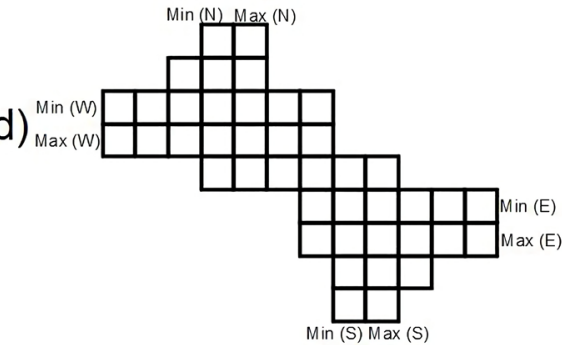

Figure 6. An element of the class: (a) $\gamma^{1,1}$, (b) $\delta^{1,1}$, (c) $\Delta^{1,1}$, (d) $\chi^{1,1}$.

or

4)

$$
\max (W) \text { to } \max (S)
$$

and $\max (N)$ to the corner cell in $S E$

Corollary 1. If $P$ satisfies the conditions of Theorem 2, then $P$ is in the class $\gamma_{2 L}^{1,1}$ and hence it is in the class $\alpha_{2 L}^{1,1}$ (see Figure 7).

Theorem 2. Let $P$ be a convex polyomino in the class $\delta^{1,1}, P$ is 2-convex if and only if there exists an $L$-path from

1)

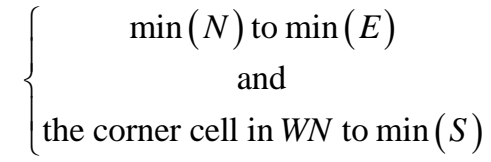

or

2)

$$
\left\{\begin{array}{c}
\max (W) \text { to } \max (S) \\
\text { and } \\
\max (N) \text { to the corner cell in } S E
\end{array}\right.
$$

or

3)

$$
\left\{\begin{array}{c}
\max (N) \text { to } \max (E) \\
\text { and } \\
\max (W) \text { to the corner cell in } S E
\end{array}\right.
$$

or

4)

$$
\left\{\begin{array}{c}
\min (W) \text { to } \min (S) \\
\text { and } \\
\text { the corner cell in } W N \text { to } \min (E)
\end{array}\right.
$$

or 


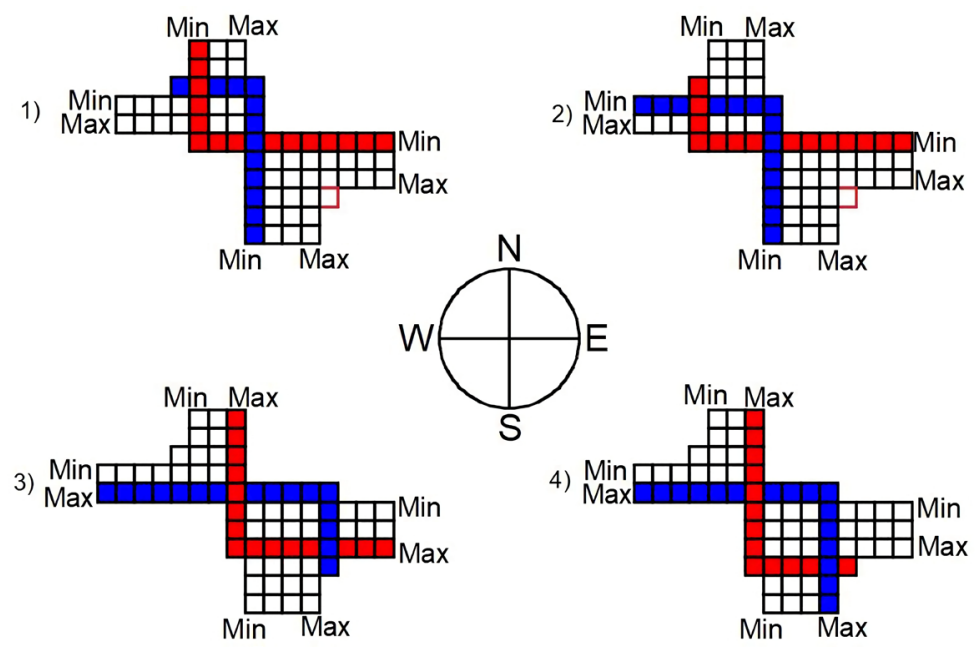

Figure 7. The four geometries in the class $\gamma_{2 L}^{1,1}$.

5)

$$
\begin{aligned}
& \max (N) \text { to } \max (E) \\
& \text { and }
\end{aligned}
$$

the corner cell in $W N$ to $\min (S)$

and

$2 L$-path starting by a south step from $\min (N)$ to the corner cell in $S E$

or

6)

$$
\min (W) \text { to } \min (S)
$$
and $\max (N)$ to the corner cell in SE and

$2 L$-path starting by a south step from the corner cell in $W N$ to $\max (S)$

or

7)

$$
\begin{aligned}
& \min (W) \text { to } \min (S) \\
& \text { and } \\
& \max (N) \text { to } \max (E) \\
& \text { and }
\end{aligned}
$$

$2 L$-path starting by a south step from the corner cell in $W N$ to the corner cell in $S E$

Corollary 2. If $P$ satisfies the conditions of Theorem 3, then $P$ is in the class $\delta_{2 L}^{1,1}$ and hence it is in the class $\alpha_{2 L}^{1,1}$ (see Figure 8).

Theorem 3. Let $P$ be a convex polyomino in the class $\Delta^{1,1}, P$ is 2 -convex if and only if there exists an $L$-path from

1)

$$
\left\{\begin{array}{c}
\min (W) \text { to } \min (S) \\
\text { and } \\
\text { the corner cell in } W N \text { to } \min (E)
\end{array}\right.
$$

or 

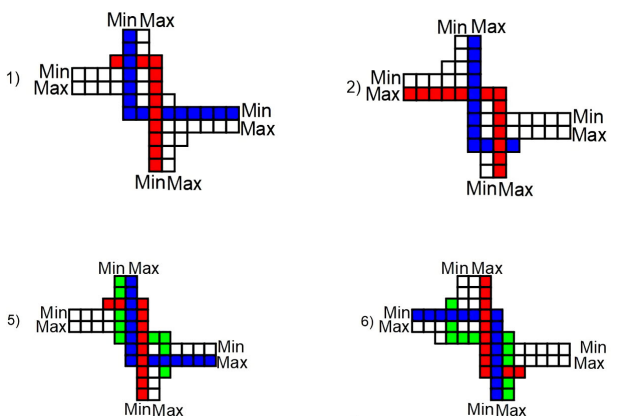
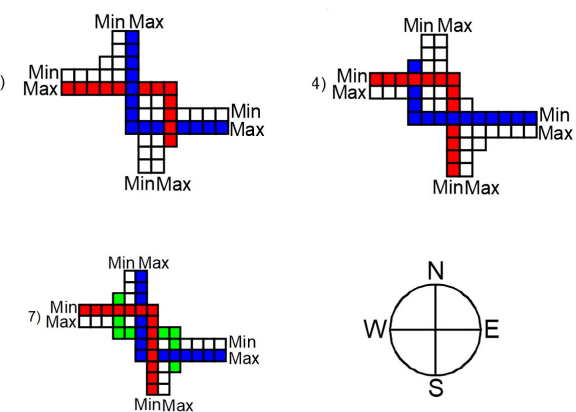

Figure 8. The seven geometries in the class $\delta_{2 L}^{1,1}$.

2)

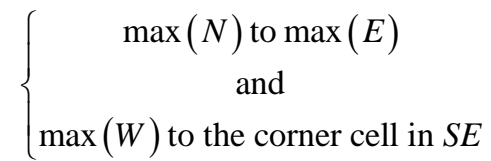

or

3)

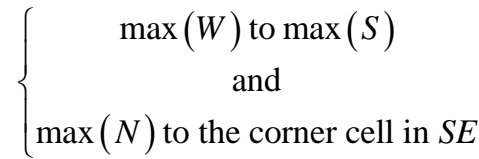

or

4)

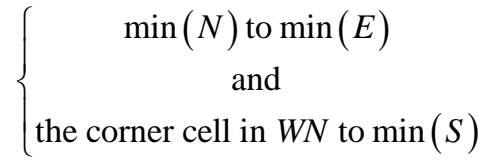

or

5)

$$
\begin{gathered}
\max (W) \text { to } \max (S) \\
\text { and }
\end{gathered}
$$

the corner cell in $W N$ to $\min (E)$

$$
\text { and }
$$

$2 L$-path starting by an east step from $\min (W)$ to the corner cell in $S E$

or

6)

$$
\left\{\begin{array}{c}
\min (N) \text { to } \min (E) \\
\text { and } \\
\max (W) \text { to the corner cell in } S E \\
\text { and } \\
2 L \text {-path starting by an east step from } \\
\text { the corner cell in } W N \text { to } \max (E)
\end{array}\right.
$$

or

$$
\begin{aligned}
& \min (N) \text { to } \min (E) \\
& \text { and } \\
& \max (W) \text { to } \max (S) \\
& \text { and }
\end{aligned}
$$

$2 L$-path starting by an east step from the corner cell in $W N$ to the corner cell in $S E$ 
Corollary 3. If $P$ satisfies the conditions of Theorem 4, then $P$ is in the class $\Delta_{2 L}^{1,1}$ and hence it is in the class $\alpha_{2 L}^{1,1}$ (see Figure 9).

Theorem 4. Let $P$ be a convex polyomino in the class $\chi^{1,1}, P$ is 2 -convex if and only if there exists an $L$-path from

1)

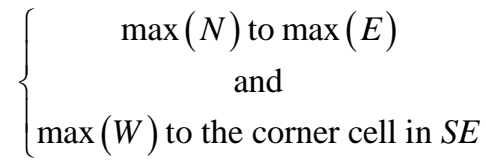

or

2)

$$
\left\{\begin{array}{c}
\min (N) \text { to } \min (E) \\
\text { and } \\
\min (W) \text { to the corner cell in } S E \\
\text { and } \\
2 L \text {-path starting by an east step from } \\
\text { the corner cell in } W N \text { to } \max (E)
\end{array}\right.
$$

or

3) $\{$ the corner cell in $W N$ to $\min (S)$

or

4)

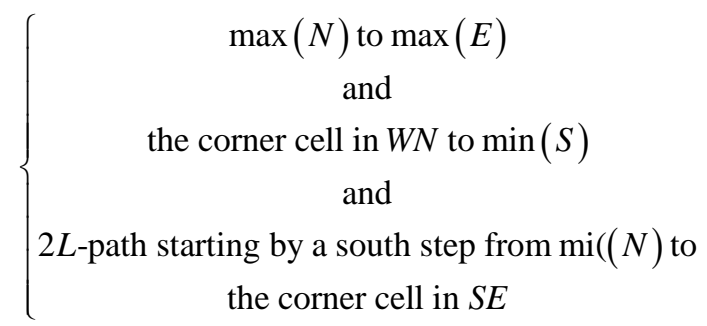

or

5)

$$
\left\{\begin{array}{c}
\max (W) \text { to } \max (S) \\
\text { and } \\
\max (N) \text { to the corner cell in } S E
\end{array}\right.
$$

or

6)

$$
\left\{\begin{array}{c}
\min (W) \text { to } \min (S) \\
\text { and } \\
\max (N) \text { to the corner cell in } S E \\
\text { and } \\
2 L \text {-path starting by a south step from } \\
\text { the corner cell in } W N \text { to } \max (S)
\end{array}\right.
$$

or

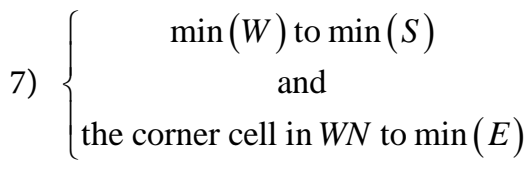

or 

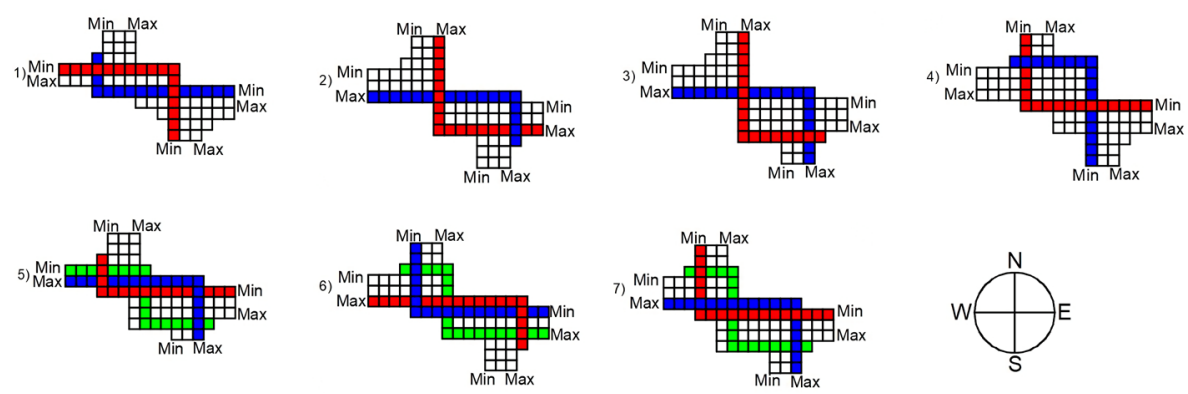

Figure 9. The seven geometries in the class $\Delta_{2 L}^{1,1}$.

8)

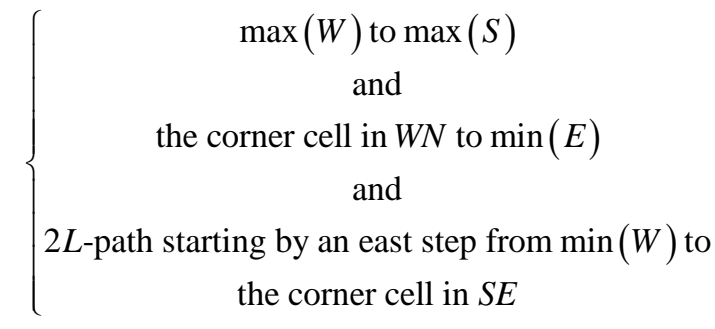

or

9)

$$
\left\{\begin{array}{c}
\max (W) \text { to } \max (S) \\
\text { and } \\
\min (N) \text { to } \min (E) \\
\text { and } \\
2 L \text {-path starting by an east step from } \\
\text { the corner cell in } W N \text { to the corner cell in } S E
\end{array}\right.
$$

)

or

$$
\begin{aligned}
& \max (W) \text { to } \max (S) \\
& \text { and } \\
& \max (N) \text { to } \max (E) \\
& \text { and }
\end{aligned}
$$

10) $\{2 L$-path starting by an east step from the corner cell in $W N$ to the corner cell in $S E$

$$
\text { and }
$$

$2 L$-path starting by a south step from $\min (N)$ to the corner cell in $S E$

or

$$
\begin{aligned}
& \min (W) \text { to } \min (S) \\
& \text { and }
\end{aligned}
$$

$$
\begin{gathered}
\min (N) \text { to } \min (E) \\
\text { and }
\end{gathered}
$$

11) $2 L$-path starting by an east step from to

the corner cell in $W N$ to the corner cell in $S E$

and

$2 L$-path starting by a south step from the corner cell in $W N$ to $\max (S)$ 
or

12)

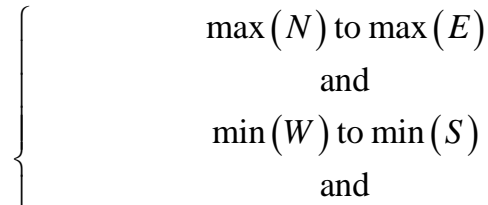

$2 L$-path starting by a south step from

the corner cell in $W N$ to the corner cell in $S E$

or

13)

$$
\begin{aligned}
& \max (W) \text { to } \max (S) \\
& \text { and } \\
& \max (N) \text { to } \max (E) \\
& \text { and }
\end{aligned}
$$

2L-path starting by a south step from

the corner cell in $W N$ to the corner cell in $S E$ and

$2 L$-path starting by an east step from $\min (W)$ to the corner cell in $S E$

or

14) $2 L$-path starting by a south step from the corner cell in $W N$ to the corner cell in $S E$ and

$2 L$-path starting by an east step from the corner cell in $W N$ to $\max (E)$

Corollary 4. If $P$ satisfies the conditions of Theorem 5 , then $P$ is in the class $\chi_{2 L}^{1,1}$ and hence it is in the class $\alpha_{2 L}^{1,1}$ (see Figure 10 and Figure 11).

\section{HV-Convex Polyominoes}

Assume that $H, V$ denote strictly positive row and column sum vectors. We also assume that $\sum_{i} h_{i}=\sum_{j} v_{j}$, since otherwise $(H, V)$ do not have a realization.

The idea of Chrobak and Dürr [8] for reconstructing an $H V$-convex polyomino is to impose convexity on the four corner regions outside of the polyomino.

An object $A$ is called an upper-left corner region if $(i+1, j) \in A$ or $(i, j+1) \in A$ implies $(i, j) \in A$. In an analogous way other corner regions can be defined. Let $\bar{P}$ be the complement of $P$. The definition of $H V$-convex polyominoes directly implies the following lemma.

Lemma 1. $P$ is an $H V$-convex polyomino if and only if $\bar{P}=A \cup B \cup C \cup D$, where $A, B, C, D$ are disjoint corner regions (upper-left, upper-right, lower-left and lower-right, respectively) such that 1$)(i-1, j-1) \in A$ implies $(i, j)$ not in $D$, and 2$) \quad(i-1, j+1) \in B$ implies $(i, j) \notin C$. 

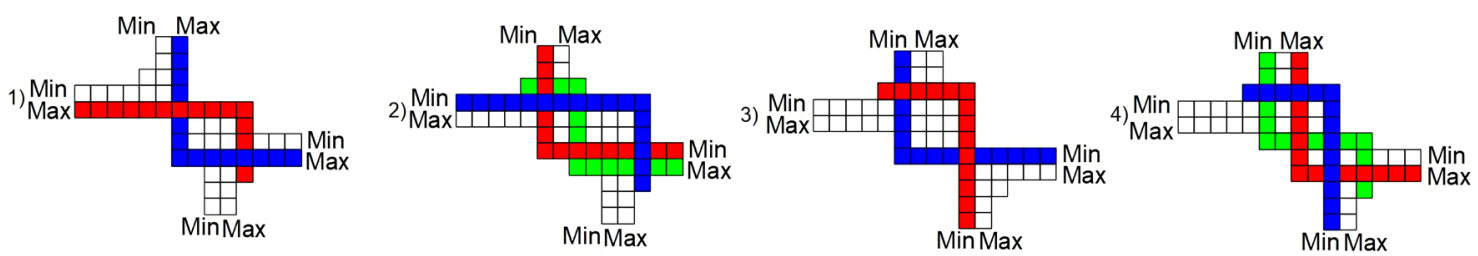

5)
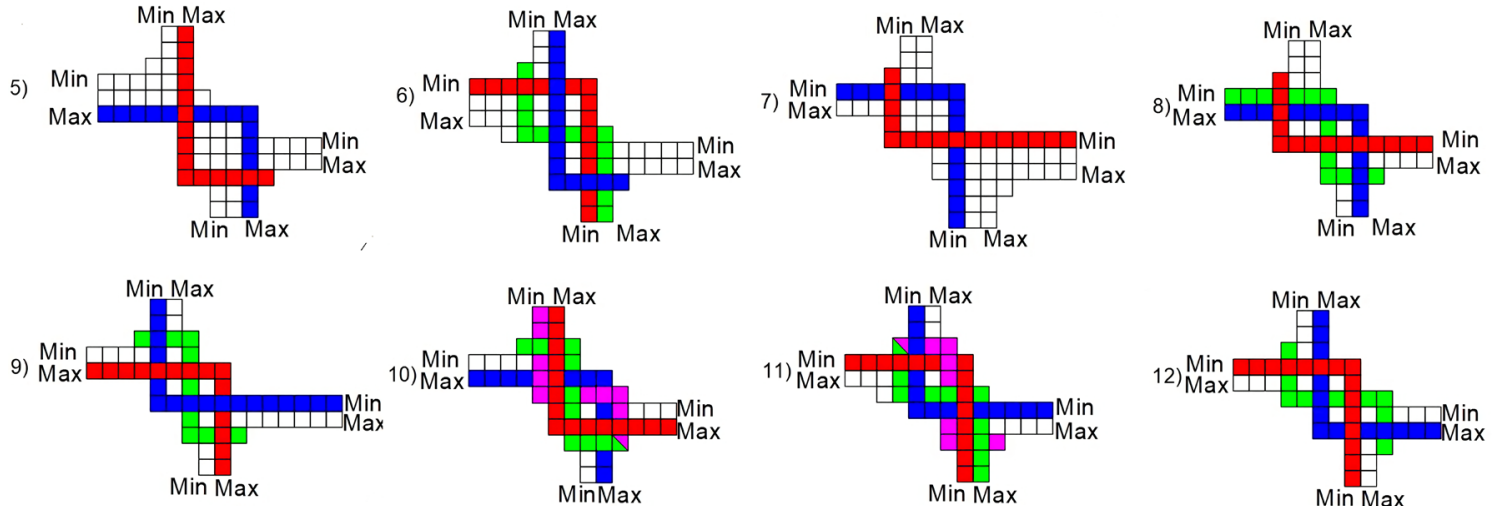

Figure 10. The first twelve geometries in the class $\chi_{2 L}^{1,1}$.
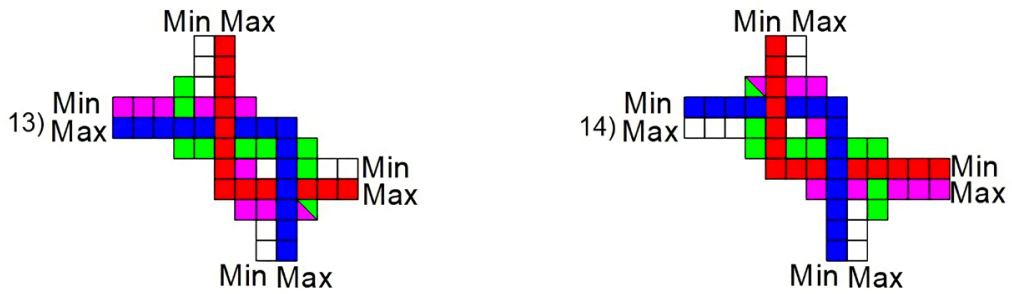

Figure 11. The last two geometries in the class $\chi_{2 L}^{1,1}$.

Given an $H V$-convex polyomino $P$ and two row indices $1 \leq k, l \leq m . P$ is anchored at $(k, l)$ if $(k, 1),(l, n) \in P$. The idea of Chrobak and Dürr is, given $(H, V)$, to reconstruct a 2SAT expression (a boolean expression in conjunctive normal form with at most two literals in each clause) $F_{k, l}(H, V)$ with the property that $F_{k, l}(H, V)$ is satisfiable iff there is an $H V$-convex polyomino realization $P$ of $(H, V)$ that is anchored at $(k, l) . F_{k, l}(H, V)$ consists of several sets of clauses, each set expressing a certain property: "Corners" (Cor), "Disjointness" (Dis), "Connectivity" (Con), "Anchors" (Anc), "Lower bound on column sums" ( $L B C)$ and "Upper bound on row sums" (UBR).

$$
\text { Cor }=\wedge_{i, j}\left\{\begin{array}{llll}
A_{i, j} \Rightarrow A_{i-1, j} & B_{i, j} \Rightarrow B_{i-1, j} & C_{i, j} \Rightarrow C_{i+1, j} & D_{i, j} \Rightarrow D_{i+1, j} \\
A_{i, j} \Rightarrow A_{i, j-1} & B_{i, j} \Rightarrow B_{i, j+1} & C_{i, j} \Rightarrow C_{i, j-1} & D_{i, j} \Rightarrow D_{i, j+1}
\end{array}\right\}
$$

The set of clauses Cor means that the corners are convex, that is for the corner $A$ if the cell $(i, j)$ belongs to $A$ then cells $(i-1, j)$ and $(i, j-1)$ belong also to $A$. Similarly for corners $B, C$, and $D$.

$$
\text { Dis }=\wedge_{i, j}\left\{X_{i, j} \Rightarrow \bar{Y}_{i, j} \text { : for symbols } X, Y \in\{A, B, C, D\}, X \neq Y\right\}
$$

The set of clauses Dis means that all four corners are pairwise disjoint, that is $X \cap Y=\varnothing$ for $X, Y \in\{A, B, C, D\}$. 


$$
\text { Con }=\wedge_{i, j}\left\{A_{i, j} \Rightarrow \bar{D}_{i+1, j+1} \quad B_{i, j} \Rightarrow \bar{C}_{i+1, j-1}\right\}
$$

The set of clauses Con means that if the cell $(i, j)$ belongs to $A$ then the cell $(i+1, j+1)$ does not belong to $D$, and similarly if the cell $(i, j)$ belongs to $B$ then the cell $(i+1, j-1)$ does not belong to $C$.

$$
\text { Anc }=\left\{\bar{A}_{k, 1} \wedge \bar{B}_{k, 1} \wedge \bar{C}_{k, 1} \wedge \bar{D}_{k, 1} \wedge \bar{A}_{l, n} \wedge \bar{B}_{l, n} \wedge \bar{C}_{l, n} \wedge \bar{D}_{l, n}\right\}
$$

The set of clauses Anc means that we fix two cells on the west and east feet of the polyomino $P$, for $k, l=1, \cdots, m$ the first one at the position $(k, 1)$ and the second one at the position $(l, n)$.

$$
L B C=\wedge_{i, j}\left\{\begin{array}{ll}
A_{i, j} \Rightarrow \bar{C}_{i+v_{j}, j} & A_{i, j} \Rightarrow \bar{D}_{i+v_{j}, j} \\
B_{i, j} \Rightarrow \bar{C}_{i+v_{j}, j} & B_{i, j} \Rightarrow \bar{D}_{i+v_{j}, j}
\end{array}\right\} \wedge \wedge_{j}\left\{\begin{array}{ll}
\bar{C}_{v_{j}, j} & \bar{D}_{v_{j}, j}
\end{array}\right\}
$$

The set of clauses $L B C$ implies that for each column $j$, we have that $\sum_{i} P_{i, j} \geq v_{j}$.

$$
U B R=\wedge_{j}\left\{\begin{array}{cc}
\wedge_{i \leq \min \{k, l\}} \bar{A}_{i, j} \Rightarrow B_{i, j+h_{i}} & \wedge_{k \leq i \leq l} \bar{C}_{i, j} \Rightarrow B_{i, j+h_{i}} \\
\wedge_{I \leq i \leq k} \bar{A}_{i, j} \Rightarrow D_{i, j+h_{i}} & \wedge_{\max \{k, l\} \leq i} \bar{C}_{i, j} \Rightarrow D_{i, j+h_{i}}
\end{array}\right\}
$$

The set of clauses $U B R$ implies that for each row $i$, we have that $\sum_{j} P_{i, j} \leq h_{i}$. Define $F_{k, l}(H, V)=C o r \wedge D i s \wedge C o n \wedge A n c \wedge L B C \wedge U B R$. All literals with indices outside the set $\{1, \cdots, m\} \times\{1, \cdots, n\}$ are assumed to have value 1 .

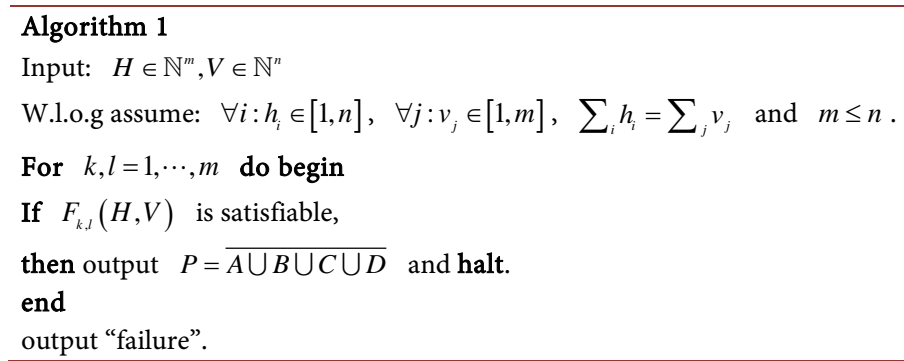

The following theorem allows to link the existence of $H V$-convex solution and the evaluation of $F_{k, l}(H, V)$. The crucial part of this algorithm comes from the constraints on the two sets of clauses $L B C$ and $U B R$.

Theorem 5 (Chrobak, Durr). $F_{k, l}(H, V)$ is satisfiable if and only if $(H, V)$ have a realization $P$ that is an $H V$-convex polyomino anchored at $(k, l)$.

Each formula $F_{k, l}(H, V)$ has size $O(m n)$ and can be computed in time $O(m n)$. Since 2SAT can be solved in linear time see [11] [12], Chrobak and Dürr give the following result.

Theorem 6 (Chrobak, Durr). Algorithm 1 solves the reconstruction problem for $H V$-convex polyominoes in time $O\left(m n \min \left(m^{2}, n^{2}\right)\right)$.

\section{Reconstruction of 2-Convex Polyominoes in $\alpha_{2 L}^{1,1}$}

The present section uses the theoretical material of the previous sections in order to reconstruct 2-convex polyominoes in the class $\alpha_{2 L}^{1,1}$. Some modifications are made to the reconstruction algorithm of Chrobak and Dürr for $H V$-convex 
polyominoes in order to impose our geometries.

Finally, by defining an horizontal symmetry $S_{H}$, we show how to reconstruct $\mathrm{P}$ in the class $\beta_{2 L}^{1,1}$.

\subsection{Clauses for the Class $\gamma_{2 L}^{1,1}$}

We code by a 2SAT formula the four geometries that characterize all 2-convex polyominoes in the class $\gamma_{2 L}^{1,1}$ in order to reconstruct them.

$$
\begin{aligned}
& \text { Pos }=\left\{C_{(\max (W)+1,1)} \wedge C_{(m, \max (N))} \wedge A_{1,1} \wedge D_{m, n}\right\}
\end{aligned}
$$

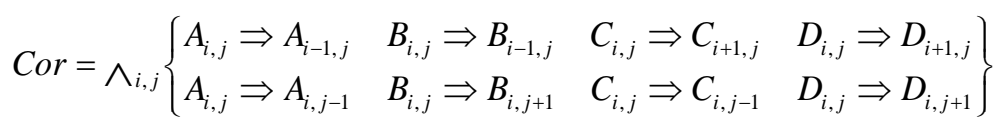

$$
\begin{aligned}
& \text { Dis }=\wedge_{i, j}\left\{X_{i, j} \Rightarrow \bar{Y}_{i, j} \text { : for symbols } X, Y \in\{A, B, C, D\}, X \neq Y\right\} \\
& \text { Con }=\wedge_{i, j}\left\{A_{i, j} \Rightarrow \bar{D}_{i+1, j+1} \quad B_{i, j} \Rightarrow \bar{C}_{i+1, j-1}\right\} \\
& A n C=\left\{\begin{array}{c}
\bar{A}_{\min (W), 1} \wedge \bar{A}_{\max (W)+1, n} \wedge \bar{B}_{\min (W), 1} \wedge \bar{B}_{\max (W)+1, n} \wedge \\
\bar{C}_{\min (W), 1} \wedge \bar{C}_{\max (W)+1, n} \wedge \bar{D}_{\min (W), 1} \wedge \bar{D}_{\max (W)+1, n} \wedge \\
\bar{A}_{1, \min (N)} \wedge \bar{A}_{m, \max (N)+1} \wedge \bar{B}_{1, \min (N)} \wedge \bar{B}_{m, \max (N)+1} \wedge \\
\bar{C}_{1, \min (N)} \wedge \bar{C}_{m, \max (N)+1} \wedge \bar{D}_{1, \min (N)} \wedge \bar{D}_{m, \max (N)+1} \wedge \\
\bar{A}_{\max (W), 1} \wedge \bar{A}_{\max (E), n} \wedge \bar{B}_{\max (W), 1} \wedge \bar{B}_{\max (E), n} \wedge \\
\bar{C}_{\max (W), 1} \wedge \bar{C}_{\max (E), n} \wedge \bar{D}_{\max (W), 1} \wedge \bar{D}_{\max (E), n} \wedge \\
\bar{A}_{1, \max (N)} \wedge \bar{A}_{m, \max (S)} \wedge \bar{B}_{1, \max (N)} \wedge \bar{B}_{m, \max (S)} \wedge \\
\bar{C}_{1, \max (N)} \wedge \bar{C}_{m, \max (S)} \wedge \bar{D}_{1, \max (N)} \wedge \bar{D}_{m, \max (S)}
\end{array}\right\} \\
& L B C=\wedge_{i}\left\{\begin{array}{cc}
\wedge_{j<\min (N)} A_{i, j} \Rightarrow \bar{C}_{i+v_{j}, j} & \wedge_{\max (N)+1 \leq j \leq \max (S)} B_{i, j} \Rightarrow \bar{C}_{i+v_{j}, j} \\
\wedge_{\min (N) \leq j \leq \max (N)} C_{i+v_{j}, j} \Rightarrow \bar{A}_{i, j} & \wedge_{j>\max (S)} B_{i, j} \Rightarrow \bar{D}_{i+v_{j}, j} \\
\wedge_{\max (N)<j<\max (N)+1} B_{i, j} \Rightarrow \bar{C}_{i+v_{j}, j} &
\end{array}\right\} \\
& \wedge \wedge_{j}\left\{\bar{C}_{v_{j}, j} \quad \bar{D}_{v_{j}, j}\right\} \\
& U B R=\wedge_{j}\left\{\begin{array}{cc}
\wedge_{i<\max (W)+1} \bar{A}_{i, j} \Rightarrow B_{i, j+h_{i}} & \wedge_{\min (W) \leq i \leq \max (W)} \bar{A}_{i, j} \Rightarrow D_{i, j+h_{i}} \\
\wedge_{\max (W)+1 \leq i \leq \max (E)} \bar{B}_{i, j+h_{i}} \Rightarrow A_{i, j} & \wedge_{i>\max (W)} \bar{C}_{i, j} \Rightarrow D_{i, j+h_{i}} \\
\wedge_{\max (E)<i<\min (W)} \bar{A}_{i, j} \Rightarrow D_{i, j+h_{i}} &
\end{array}\right\} \\
& R E C=\left\{\bar{A}_{\min (W)-1, \min (N)-1} \wedge \bar{D}_{\max (E)+1, \max (S)+1}\right\} \\
& R E C 1=\left\{\begin{array}{c}
A_{\min (W)-1, \min (N)-2} \wedge A_{\min (W)-2, \min (N)-1} \wedge D_{\max (E)+1, \max (S)+2} \\
\wedge D_{\max (E)+2, \max (S)+1}
\end{array}\right\} \\
& G E O 1=\left\{\begin{array}{c}
\bar{A}_{\min (N), \max (W)+1} \wedge \bar{B}_{\min (N), \max (W)+1} \wedge \bar{C}_{\min (N), \max (W)+1} \\
\wedge \bar{D}_{\min (N), \max (W)+1} \wedge \bar{A}_{\min (W)-1, \max (N)+1} \wedge \bar{B}_{\min (W)-1, \max (N)+1} \\
\wedge \bar{C}_{\min (W)-1, \max (N)+1} \wedge \bar{D}_{\min (W)-1, \max (N)+1}
\end{array}\right\}
\end{aligned}
$$




$$
\begin{aligned}
& G E O 2=\left\{\begin{array}{c}
\bar{A}_{\min (W), \max (N)+1} \wedge \bar{B}_{\min (W), \max (N)+1} \wedge \bar{C}_{\min (W), \max (N)+1} \\
\wedge \bar{D}_{\min (W), \max (N)+1} \wedge \bar{A}_{\min (W)-1, \max (W)+1} \wedge \bar{B}_{\min (W)-1, \max (W)+1} \\
\wedge \bar{C}_{\min (W)-1, \max (W)+1} \wedge \bar{D}_{\min (W)-1, \max (W)+1}
\end{array}\right\} \\
& \text { GEO3 }=\left\{\begin{array}{c}
\bar{A}_{\max (E), \max (N)} \wedge \bar{B}_{\max (E), \max (N)} \wedge \bar{C}_{\max (E), \max (N)} \\
\wedge \bar{D}_{\max (E), \max (N)} \wedge \bar{A}_{\max (W), \max (S)+1} \wedge \bar{B}_{\max (W), \max (S)+1} \\
\wedge \bar{C}_{\max (W), \max (S)+1} \wedge \bar{D}_{\max (W), \max (S)+1}
\end{array}\right\} \\
& \text { GEO4 }=\left\{\begin{array}{c}
\bar{A}_{\max (W), \max (S)} \wedge \bar{B}_{\max (W), \max (S)} \wedge \bar{C}_{\max (W), \max (S)} \\
\wedge \bar{D}_{\max (W), \max (S)} \wedge \bar{A}_{\max (E)+1, \max (N)} \wedge \bar{B}_{\max (E)+1, \max (N)} \\
\wedge \bar{C}_{\max (E)+1, \max (N)} \wedge \bar{D}_{\max (E)+1, \max (N)}
\end{array}\right\}
\end{aligned}
$$

In order to reconstruct all 2-convex polyominoes in the class $\gamma_{2 L}^{1,1}$, we use the set of clauses that impose the 4 geometries.

$$
\begin{aligned}
\gamma_{2 L, G e o 1}^{1,1}(H, V)= & P \text { os } \wedge \text { Cor } \wedge \text { Dis } \wedge \text { Con } \wedge \text { Anc } \wedge L B C \wedge U B R \\
& \wedge R E C \wedge R E C 1 \wedge G E O 1 . \\
\gamma_{2 l, G e o 2}^{1,1}(H, V)= & P o s \wedge \text { Cor } \wedge \text { Dis } \wedge \text { Con } \wedge \text { Anc } \wedge L B C \wedge U B R \\
& \wedge R E C \wedge R E C 1 \wedge G E O 2 . \\
\gamma_{2 L, G e o 3}^{1,1}(H, V)= & P o s \wedge \text { Cor } \wedge \text { Dis } \wedge \text { Con } \wedge \text { Anc } \wedge L B C \wedge U B R \\
& \wedge R E C \wedge R E C 1 \wedge G E O 3 . \\
\gamma_{2 L, G e o 4}^{1,1}(H, V)= & P o s \wedge \text { Cor } \wedge \text { Dis } \wedge \text { Con } \wedge \text { Anc } \wedge L B C \wedge U B R \\
& \wedge R E C \wedge R E C 1 \wedge G E O 4 .
\end{aligned}
$$

\section{Algorithm 2}

Input: $H \in \mathbb{N}^{m}, V \in \mathbb{N}^{n}$

W.l.o.g assume: $\forall i: h_{i} \in[1, n], \forall j: v_{j} \in[1, m], \quad \sum_{i} h_{i}=\sum_{j} v_{j}$.

For $\min (W), \min (E)=1, \cdots, m$ and

$\min (N), \min (S)=1, \cdots, n$ do begin

If $\gamma_{2 L, \text { Geo } 1}^{1,1}(H, V)$ or $\gamma_{2 L, \text { Geo } 2}^{1,1}(H, V)$ or $\gamma_{2 L, \text { Geo }}^{1,1}(H, V)$ or $\gamma_{2 L, G \text { Geo }}^{1,1}(H, V)$ is satisfiable,

then output $P=\overline{A \cup B \cup C \cup D}$ and halt.

end

output "failure".

Proof. The following modifications of the original algorithm of Chrobak and Durr [8] are made in order to add the geometrical constraints of the class $\gamma_{2 L}^{1,1}$.

- The set Pos imposes the constraint of the relative positions of feet in $\gamma_{2 L}^{1,}$ with $\min (E)=\max (W)+1$ and $\min (S)=\max (N)+1$.

- The set REC implies that thes cells at the positions $(\min (W)-1, \min (N)-1)$ and $(\max (E)+1, \max (S)+1)$ are in the interior of the polyomino.

- The set REC1 implies that the cells at the prositions $(\min (W)-1, \min (N)-2),(\min (W)-2, \min (N)-1)$, $(\max (E)+1, \max (S)+2)$, and $(\max (E)+2, \max (S)+1)$ are not in the interior of the polyomino.

- The set GEO1 implies that we put a cell in the interior of the polyomino at 
the position $(\max (W)-1, \max (N)+1)$ (resp. $(\min (N), \max (W)+1)$ and then by convexity an $L$-path between $\max (W)-1$ and $\max (N)+1$ (resp. $\min (N)$ and $\max (W)+1)$. Thus we have exactly the definition of the first geometry. The set GEO2, GEO3 and GEO4 give respectively the $L$-paths of the second geometry, the third geometry and the fourth geometry.

Using the conjunction of the whole set of clauses, if one of the $\gamma_{2 L, G e 01}^{1,1}(H, V)$ or $\gamma_{2 L, G e 02}^{1,1}$ or $\gamma_{2 L, G e o 3}^{1,1}$ or $\gamma_{2 L, G e o 4}^{1,1}$ is satisfiable then we are able to reconstruct an $H V$-convex with the constraints of the subclass $\gamma_{2 L}^{1,1}$ (see Figure 12).

\subsection{Clauses for the Class $\delta_{2 L}^{1,1}$}

We code by a 2SAT formula the seven geometries that characterize all 2-convex polyominoes in the class $\delta_{2 L}^{1,1}$ in order to reconstruct them.

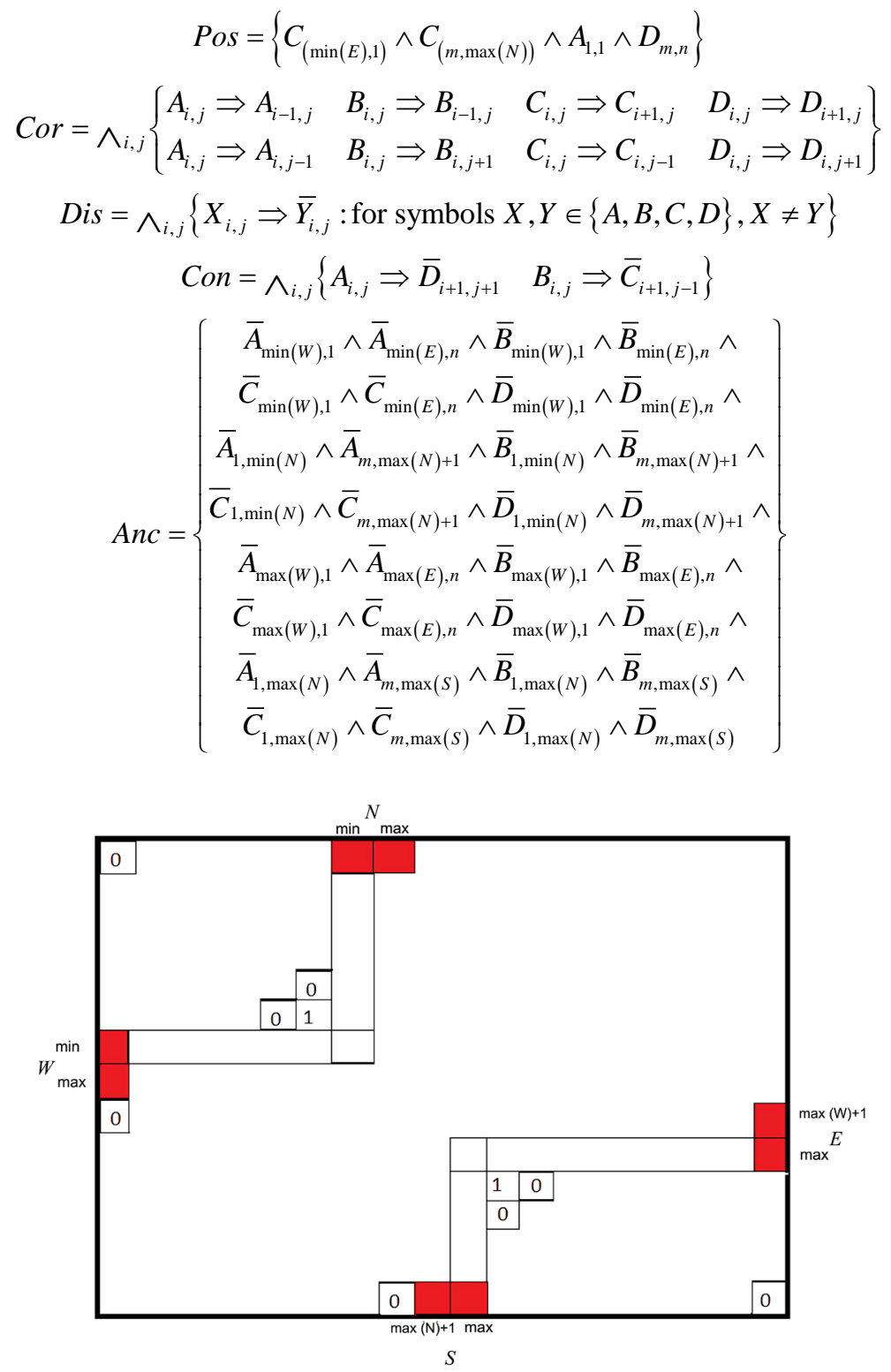

Figure 12. Pos, ANC, REC and REC1 in the class $\gamma_{2 L}^{1,1}$ 


$$
\begin{aligned}
& L B C=\wedge_{i}\left\{\begin{array}{cc}
\wedge_{j<\min (N)} A_{i, j} \Rightarrow \bar{C}_{i+v_{j}, j} & \wedge_{\max (N)+1 \leq j \leq \max (S)} B_{i, j} \Rightarrow \bar{C}_{i+v_{j}, j} \\
\wedge_{\min (N) \leq j \leq \max (N)} C_{i+v_{j}, j} \Rightarrow \bar{A}_{i, j} & \wedge_{j>\max (S)} B_{i, j} \Rightarrow \bar{D}_{i+v_{j}, j} \\
\wedge_{\max (N)<j<\max (N)+1} B_{i, j} \Rightarrow \bar{C}_{i+v_{j}, j} &
\end{array}\right\} \\
& \wedge \wedge_{j}\left\{\bar{C}_{v_{j}, j} \quad \bar{D}_{v_{j}, j}\right\} \\
& U B R=\wedge_{j}\left\{\begin{array}{cc}
\wedge_{i<\min (E)} \bar{A}_{i, j} \Rightarrow B_{i, j+h_{i}} & \wedge_{\min (W) \leq i \leq \max (W)} \bar{A}_{i, j} \Rightarrow D_{i, j+h_{i}} \\
\wedge_{\min (E) \leq i \leq \max (E)} \bar{B}_{i, j+h_{i}} \Rightarrow A_{i, j} & \wedge_{i>\max (W)} \bar{C}_{i, j} \Rightarrow D_{i, j+h_{i}} \\
\wedge_{\max (E)<i<\min (W)} \bar{A}_{i, j} \Rightarrow D_{i, j+h_{i}} &
\end{array}\right\} \\
& R E C=\left\{\bar{A}_{\min (W)-1, \min (N)-1} \wedge \bar{D}_{\max (E)+1, \max (S)+1}\right\} \\
& R E C 1=\left\{\begin{array}{c}
A_{\min (W)-1, \min (N)-2} \wedge A_{\min (W)-2, \min (N)-1} \wedge D_{\max (E)+1, \max (S)+2} \\
\wedge D_{\max (E)+2, \max (S)+1}
\end{array}\right\} \\
& G E O 1=\left\{\begin{array}{c}
\bar{A}_{\min (E), \min (N)} \wedge \bar{B}_{\min (E), \min (N)} \wedge \bar{C}_{\min (E), \min (N)} \\
\wedge \bar{D}_{\min (E), \min (N)} \wedge \bar{A}_{\min (W)-1, \max (N)+1} \wedge \bar{B}_{\min (W)-1, \max (N)+1} \\
\wedge \bar{C}_{\min (W)-1, \max (N)+1} \wedge \bar{D}_{\min (W)-1, \max (N)+1}
\end{array}\right\} \\
& \text { GEO2 }=\left\{\begin{array}{c}
\bar{A}_{\max (W), \max (S)} \wedge \bar{B}_{\max (W), \max (S)} \wedge \bar{C}_{\max (W), \max (S)} \\
\wedge \bar{D}_{\max (W), \max (S)} \wedge \bar{A}_{\max (E)+1, \max (N)} \wedge \bar{B}_{\max (E)+1, \max (N)} \\
\wedge \bar{C}_{\max (E)+1, \max (N)} \wedge \bar{D}_{\max (E)+1, \max (N)}
\end{array}\right\} \\
& \text { GEO3 }=\left\{\begin{array}{c}
\bar{A}_{\max (E), \max (N)} \wedge \bar{B}_{\max (E), \max (N)} \wedge \bar{C}_{\max (E), \max (N)} \\
\wedge \bar{D}_{\max (E), \max (N)} \wedge \bar{A}_{\max (W), \max (S)+1} \wedge \bar{B}_{\max (W), \max (S)+1} \\
\wedge \bar{C}_{\max (W), \max (S)+1} \wedge \bar{D}_{\max (W), \max (S)+1}
\end{array}\right\} \\
& \text { GEO4 }=\left\{\begin{array}{c}
\bar{A}_{\min (W), \max (N)+1} \wedge \bar{B}_{\min (W), \max (N)+1} \wedge \bar{C}_{\min (W), \max (N)+1} \\
\wedge \bar{D}_{\min (W), \max (N)+1} \wedge \bar{A}_{\min (E), \min (N)-1} \wedge \bar{B}_{\min (E), \min (N)-1} \\
\wedge \bar{C}_{\min (E), \min (N)-1} \wedge \bar{D}_{\min (E), \min (N)-1}
\end{array}\right\} \\
& \text { LGEO5 }=\left\{\begin{array}{c}
\bar{A}_{\max (E), \max (N)} \wedge \bar{B}_{\max (E), \max (N)} \wedge \bar{C}_{\max (E), \max (N)} \\
\wedge \bar{D}_{\max (E), \max (N)} \wedge \bar{A}_{\min (W)-1, \max (N)+1} \wedge \bar{B}_{\min (W)-1, \max (N)+1} \\
\wedge \bar{C}_{\min (W)-1, \max (N)+1} \wedge \bar{D}_{\min (W)-1, \max (N)+1}
\end{array}\right\} \\
& 2 L G E O 5=\left\{\begin{array}{c}
C_{i, \min (N)} \Rightarrow \bar{B}_{i-1, \max (S)+1} \wedge_{i>1} \\
\bar{X}_{\max (W)+1, \min (N)} \wedge \bar{X}_{\min (E)-1, \max (S)+1}, \forall X \in\{A, B, C, D\}
\end{array}\right\} \\
& \text { LGEO6 }=\left\{\begin{array}{c}
\bar{A}_{\max (E)+1, \max (N)} \wedge \bar{B}_{\max (E)+1, \max (N)} \wedge \bar{C}_{\max (E)+1, \max (N)} \\
\wedge \bar{D}_{\max (E)+1, \max (N)} \wedge \bar{A}_{\min (W), \max (N)+1} \wedge \bar{B}_{\min (W), \max (N)+1} \\
\wedge \bar{C}_{\min (W), \max (N)+1} \wedge \bar{D}_{\min (W), \max (N)+1}
\end{array}\right\} \\
& 2 L G E O 6=\left\{\begin{array}{c}
C_{i, \min (N)-1} \Rightarrow \bar{B}_{i-1, \max (S)} \wedge_{i>1} \\
\bar{X}_{\max (W)+1, \min (N)-1} \wedge \bar{X}_{\min (E)-1, \max (S)}, \forall X \in\{A, B, C, D\}
\end{array}\right\}
\end{aligned}
$$




$$
\begin{gathered}
\text { LGEO7 }=\left\{\begin{array}{c}
\bar{A}_{\min (W), \max (N)+1} \wedge \bar{B}_{\min (W), \max (N)+1} \wedge \bar{C}_{\min (W), \max (N)+1} \\
\wedge \bar{D}_{\min (W), \max (N)+1} \wedge \bar{A}_{\max (E), \max (N)} \wedge \bar{B}_{\max (E), \max (N)} \wedge \bar{C}_{\max (E), \max (N)} \wedge \bar{D}_{\max (E), \max (N)}
\end{array}\right\} \\
\text { 2LGEO7 }=\left\{\begin{array}{c}
C_{i, \min (N)-1} \Rightarrow \bar{B}_{i-1, \max (S)+1} \wedge \wedge_{i>1} \\
\bar{X}_{\max (W)+1, \min (N)-1} \wedge \bar{X}_{\min (E)-1, \max (S)+1}, \forall X \in\{A, B, C, D\}
\end{array}\right\}
\end{gathered}
$$

In order to reconstruct all 2-convex polyominoes in the class $\delta_{2 L}^{1,1}$, we use the set of clauses that impose the 7 geometries.

$$
\begin{aligned}
& \delta_{2 L, G e o 1}^{1,1}(H, V)=P o s \wedge C o r \wedge D i s \wedge C o n \wedge A n c \wedge L B C \wedge U B R \\
& \wedge R E C \wedge R E C 1 \wedge G E O 1 . \\
& \delta_{2 L, G e o 2}^{1,1}(H, V)=P o s \wedge \text { Cor } \wedge \text { Dis } \wedge \text { Con } \wedge A n c \wedge L B C \wedge U B R \\
& \wedge R E C \wedge R E C 1 \wedge G E O 2 . \\
& \delta_{2 L, \text { Geo3 }}^{1,1}(H, V)=P \text { os } \wedge \text { Cor } \wedge \text { Dis } \wedge \text { Con } \wedge \text { Anc } \wedge L B C \wedge U B R \\
& \wedge R E C \wedge R E C 1 \wedge G E O 3 \text {. } \\
& \delta_{2 L, G e o 4}^{1,1}(H, V)=P o s \wedge \text { Cor } \wedge \text { Dis } \wedge \text { Con } \wedge \text { Anc } \wedge L B C \wedge U B R \\
& \wedge R E C \wedge R E C 1 \wedge G E O 4 \text {. } \\
& \delta_{2 L, L G e o 5,2 L G E O 5}^{1,1}(H, V)=P o s \wedge \text { Cor } \wedge \text { Dis } \wedge \text { Con } \wedge A n c \wedge L B C \wedge U B R \\
& \wedge R E C \wedge R E C 1 \wedge L G E O 5 \wedge 2 L G E O 5 . \\
& \delta_{2 L, L G e o 6,2 L G E O 6}^{1,1}(H, V)=P o s \wedge C o r \wedge \text { Dis } \wedge \text { Con } \wedge \text { Anc } \wedge L B C \wedge U B R \\
& \wedge R E C \wedge R E C 1 \wedge L G E O 6 \wedge 2 L G E O 6 \text {. } \\
& \delta_{2 L, L G e o 7,2 L G E O 7}^{1,1}(H, V)=P o s \wedge C o r \wedge D i s \wedge C o n \wedge A n c \wedge L B C \wedge U B R \\
& \wedge R E C \wedge R E C 1 \wedge L G E O 7 \wedge 2 L G E O 7 .
\end{aligned}
$$

\section{Algorithm 3}

Input: $H \in \mathbb{N}^{m}, V \in \mathbb{N}^{n}$

W.l.o.g assume: $\forall i: h_{i} \in[1, n], \forall j: v_{j} \in[1, m], \quad \sum_{i} h_{i}=\sum_{j} v_{j}$.

For $\min (W), \min (E)=1, \cdots, m$ and

$\min (N), \min (S)=1, \cdots, n$ do begin

If $\delta_{2 L, G \text { Geo }}^{1,1}(H, V)$ or $\delta_{2 L, G e o 2}^{1,1}(H, V)$ or $\ldots$ or $\delta_{2 L, L G e 07,2 L G E O 7}^{1,1}(H, V)$ is satisfiable,

then output $P=\overline{A \cup B \cup C \cup D}$ and halt.

end

output "failure".

\subsection{Clauses for the Class $\Delta_{2 L}^{1,1}$}

We code by a 2SAT formula the seven geometries that characterize all 2-convex polyominoes in the class $\Delta_{2 L}^{1,1}$ in order to reconstruct them.

$$
\begin{gathered}
\text { Pos }=\left\{C_{(\max (W)+1,1)} \wedge C_{(m, \max (N))} \wedge A_{1,1} \wedge D_{m, n}\right\} \\
\text { Cor }=\wedge_{i, j}\left\{\begin{array}{llll}
A_{i, j} \Rightarrow A_{i-1, j} & B_{i, j} \Rightarrow B_{i-1, j} & C_{i, j} \Rightarrow C_{i+1, j} & D_{i, j} \Rightarrow D_{i+1, j} \\
A_{i, j} \Rightarrow A_{i, j-1} & B_{i, j} \Rightarrow B_{i, j+1} & C_{i, j} \Rightarrow C_{i, j-1} & D_{i, j} \Rightarrow D_{i, j+1}
\end{array}\right\}
\end{gathered}
$$




$$
\begin{aligned}
& \text { Dis }=\wedge_{i, j}\left\{X_{i, j} \Rightarrow \bar{Y}_{i, j} \text { : for symbols } X, Y \in\{A, B, C, D\}, X \neq Y\right\} \\
& \text { Con }=\wedge_{i, j}\left\{A_{i, j} \Rightarrow \bar{D}_{i+1, j+1} \quad B_{i, j} \Rightarrow \bar{C}_{i+1, j-1}\right\} \\
& A n C=\left\{\begin{array}{c}
\bar{A}_{\min (W), 1} \wedge \bar{A}_{\max (W)+1, n} \wedge \bar{B}_{\min (W), 1} \wedge \bar{B}_{\max (W)+1, n} \wedge \\
\bar{C}_{\min (W), 1} \wedge \bar{C}_{\max (W)+1, n} \wedge \bar{D}_{\min (W), 1} \wedge \bar{D}_{\max (W)+1, n} \wedge \\
\bar{A}_{1, \min (N)} \wedge \bar{A}_{m, \min (S)} \wedge \bar{B}_{1, \min (N)} \wedge \bar{B}_{m, \min (S)} \wedge \\
\bar{C}_{1, \min (N)} \wedge \bar{C}_{m, \min (S)} \wedge \bar{D}_{1, \min (N)} \wedge \bar{D}_{m, \min (S)} \wedge \\
\bar{A}_{\max (W), 1} \wedge \bar{A}_{\max (E), n} \wedge \bar{B}_{\max (W), 1} \wedge \bar{B}_{\max (E), n} \wedge \\
\bar{C}_{\max (W), 1} \wedge \bar{C}_{\max (E), n} \wedge \bar{D}_{\max (W), 1} \wedge \bar{D}_{\max (E), n} \wedge \\
\bar{A}_{1, \max (N)} \wedge \bar{A}_{m, \max (S)} \wedge \bar{B}_{1, \max (N)} \wedge \bar{B}_{m, \max (S)} \wedge \\
\bar{C}_{1, \max (N)} \wedge \bar{C}_{m, \max (S)} \wedge \bar{D}_{1, \max (N)} \wedge \bar{D}_{m, \max (S)}
\end{array}\right\} \\
& L B C=\wedge_{i}\left\{\begin{array}{cc}
\wedge_{j<\min (N)} A_{i, j} \Rightarrow \bar{C}_{i+v_{j}, j} & \wedge_{\min (S) \leq j \leq \max (S)} B_{i, j} \Rightarrow \bar{C}_{i+v_{j}, j} \\
\wedge_{\min (N) \leq j \leq \max (N)} C_{i+v_{j}, j} \Rightarrow \bar{A}_{i, j} & \wedge_{j>\max (S)} B_{i, j} \Rightarrow \bar{D}_{i+v_{j}, j} \\
\wedge_{\max (N)<j<\min (S)} B_{i, j} \Rightarrow \bar{C}_{i+v_{j}, j} &
\end{array}\right\} \\
& \wedge \wedge_{j}\left\{\bar{C}_{v_{j}, j} \quad \bar{D}_{v_{j}, j}\right\} \\
& U B R=\wedge_{j}\left\{\begin{array}{cc}
\wedge_{i<\max (W)+1} \bar{A}_{i, j} \Rightarrow B_{i, j+h_{i}} & \wedge_{\min (W) \leq i \leq \max (W)} \bar{A}_{i, j} \Rightarrow D_{i, j+h_{i}} \\
\wedge_{\max (W)+1 \leq i \leq \max (E)} \bar{B}_{i, j+h_{i}} \Rightarrow A_{i, j} & \wedge_{i>\max (W)} \bar{C}_{i, j} \Rightarrow D_{i, j+h_{i}} \\
\wedge_{\max (E)<i<\min (W)} \bar{A}_{i, j} \Rightarrow D_{i, j+h_{i}} &
\end{array}\right\} \\
& R E C=\left\{\bar{A}_{\min (W)-1, \min (N)-1} \wedge \bar{D}_{\max (E)+1, \max (S)+1}\right\} \\
& R E C 1=\left\{\begin{array}{c}
A_{\min (W)-1, \min (N)-2} \wedge A_{\min (W)-2, \min (N)-1} \wedge D_{\max (E)+1, \max (S)+2} \\
\wedge D_{\max (E)+2, \max (S)+1}
\end{array}\right\} \\
& G E O 1=\left\{\begin{array}{c}
\bar{A}_{\min (W), \min (S)} \wedge \bar{B}_{\min (W), \min (S)} \wedge \bar{C}_{\min (W), \min (S)} \\
\wedge \bar{D}_{\min (W), \min (S)} \wedge \bar{A}_{\max (W)+1, \min (N)-1} \wedge \bar{B}_{\max (W)+1, \min (N)-1} \\
\wedge \bar{C}_{\max (W)+1, \min (N)-1} \wedge \bar{D}_{\max (W)+1, \min (N)-1}
\end{array}\right\} \\
& G E O 2=\left\{\begin{array}{c}
\bar{A}_{\max (E), \max (N)} \wedge \bar{B}_{\max (E), \max (N)} \wedge \bar{C}_{\max (E), \max (N)} \\
\wedge \bar{D}_{\max (E), \max (N)} \wedge \bar{A}_{\max (W), \max (S)+1} \wedge \bar{B}_{\max (W), \max (S)+1} \\
\wedge \bar{C}_{\max (W), \max (S)+1} \wedge \bar{D}_{\max (W), \max (S)+1}
\end{array}\right\} \\
& \text { GEO3 }=\left\{\begin{array}{c}
\bar{A}_{\max (E), \max (S)} \wedge \bar{B}_{\max (E), \max (S)} \wedge \bar{C}_{\max (E), \max (S)} \\
\wedge \bar{D}_{\max (E), \max (S)} \wedge \bar{A}_{\max (E)+1, \max (N)} \wedge \bar{B}_{\max (E)+1, \max (N)} \\
\wedge \bar{C}_{\max (E)+1, \max (N)} \wedge \bar{D}_{\max (E)+1, \max (N)}
\end{array}\right\} \\
& G E O 4=\left\{\begin{array}{c}
\bar{A}_{\max (W)+1, \min (N)} \wedge \bar{B}_{\max (W)+1, \min (N)} \wedge \bar{C}_{\max (W)+1, \min (N)} \\
\wedge \bar{D}_{\max (W)+1, \min (N)} \wedge \bar{A}_{\min (W)-1, \min (S)} \wedge \bar{B}_{\min (W)-1, \min (S)} \\
\wedge \bar{C}_{\min (W)-1, \min (S)} \wedge \bar{D}_{\min (W)-1, \min (S)}
\end{array}\right\}
\end{aligned}
$$




$$
\begin{aligned}
& \text { LGEO5 }=\left\{\begin{array}{c}
\bar{A}_{\max (W), \max (S)} \wedge \bar{B}_{\max (W), \max (S)} \wedge \bar{C}_{\max (W), \max (S)} \\
\wedge \bar{D}_{\max (W), \max (S)} \wedge \bar{A}_{\min (W)-1, \max (W)+1} \wedge \bar{B}_{\min (W)-1, \max (W)+1} \\
\wedge \bar{C}_{\min (W)-1, \max (W)+1} \wedge \bar{D}_{\min (W)-1, \max (W)+1}
\end{array}\right\} \\
& 2 L G E O 5=\left\{\begin{array}{c}
B_{\min (W), j} \Rightarrow \bar{C}_{\max (E)+1, j-1} \wedge \wedge_{j>1} \\
\bar{X}_{\min (W), \max (N)+1} \wedge \bar{X}_{\max (E)+1, \min (S)-1}, \forall X \in\{A, B, C, D\}
\end{array}\right\} \\
& \text { LGEO6 }=\left\{\begin{array}{c}
\bar{A}_{\max (W)+1, \min (N)} \wedge \bar{B}_{\max (W)+1, \min (N)} \wedge \bar{C}_{\max (W)+1, \min (N)} \bar{D}_{\max (W)+1, \min (N)} \wedge \bar{A}_{\max (W), \max (S)+1} \wedge \bar{B}_{\max (W), \max (S)+1} \\
\wedge \bar{C}_{\max (W), \max (S)+1} \wedge \bar{D}_{\max (W), \max (S)+1}
\end{array}\right\} \\
& \text { 2LGEO6 }=\left\{\begin{array}{c}
B_{\min (W)-1, j} \Rightarrow \bar{C}_{\max (E), j-1} \wedge_{j>1} \\
\bar{X}_{\min (W)-1, \max (N)+1} \wedge \bar{X}_{\max (E), \min (S)-1}, \forall X \in\{A, B, C, D\}
\end{array}\right\} \\
& \text { LGEO7 }=\left\{\begin{array}{c}
\bar{A}_{\max (W)+1, \min (N)} \wedge \bar{B}_{\max (W)+1, \min (N)} \wedge \bar{C}_{\max (W)+1, \min (N)} \\
\wedge \bar{D}_{\max (W)+1, \min (N)} \wedge \bar{A}_{\max (W), \max (S)} \wedge \bar{B}_{\max (W), \max (S)} \\
\wedge \bar{C}_{\max (W), \max (S)} \wedge \bar{D}_{\max (W), \max (S)}
\end{array}\right\} \\
& 2 L G E O 7=\left\{\begin{array}{c}
B_{\min (W)-1, j} \Rightarrow \bar{C}_{\max (E)+1, j-1} \wedge_{j>1} \\
\bar{X}_{\min (W)-1, \max (N)+1} \wedge \bar{X}_{\max (E)+1, \min (S)-1}, \forall X \in\{A, B, C, D\}
\end{array}\right\}
\end{aligned}
$$

In order to reconstruct all 2-convex polyominoes in the class $\Delta_{2 L}^{1,1}$, we use the set of clauses that impose the 7 geometries.

$$
\begin{aligned}
& \Delta_{2 L, G e o 1}^{1,1}(H, V)=P o s \wedge \text { Cor } \wedge \text { Dis } \wedge \text { Con } \wedge A n C \wedge L B C \wedge U B R \\
& \wedge R E C \wedge R E C 1 \wedge G E O 1 . \\
& \Delta_{2 L, G e o 2}^{1,1}(H, V)=P o s \wedge C o r \wedge D i s \wedge C o n \wedge A n c \wedge L B C \wedge U B R \\
& \wedge R E C \wedge R E C 1 \wedge G E O 2 . \\
& \Delta_{2 L, \text { Geo3 }}^{1,1}(H, V)=P \text { os } \wedge \text { Cor } \wedge \text { Dis } \wedge \text { Con } \wedge \text { Anc } \wedge L B C \wedge U B R \\
& \wedge R E C \wedge R E C 1 \wedge G E O 3 . \\
& \Delta_{2 L, G e o 4}^{1,1}(H, V)=P o s \wedge \text { Cor } \wedge \text { Dis } \wedge \text { Con } \wedge \text { Anc } \wedge L B C \wedge U B R \\
& \wedge R E C \wedge R E C 1 \wedge G E O 4 . \\
& \Delta_{2 L, L G e 05,2 L G E O 5}^{1,1}(H, V)=P o s \wedge C o r \wedge \text { Dis } \wedge \text { Con } \wedge \text { Anc } \wedge L B C \wedge U B R \\
& \wedge R E C \wedge R E C 1 \wedge L G E O 5 \wedge 2 L G E O 5 . \\
& \Delta_{2 L, L G e o 6,2 L G E O 6}^{1,1}(H, V)=P o s \wedge C o r \wedge D i s \wedge C o n \wedge A n c \wedge L B C \wedge U B R \\
& \wedge R E C \wedge R E C 1 \wedge L G E O 6 \wedge 2 L G E O 6 . \\
& \Delta_{2 L, L G e 07,2 L G E O 7}^{1,1}(H, V)=P o s \wedge C o r \wedge D i s \wedge C o n \wedge A n c \wedge L B C \wedge U B R \\
& \wedge R E C \wedge R E C 1 \wedge L G E O 7 \wedge 2 L G E O 7 .
\end{aligned}
$$

\section{Algorithm 4}

Input: $H \in \mathbb{N}^{m}, V \in \mathbb{N}^{n}$

W.l.o.g assume: $\forall i: h_{i} \in[1, n], \forall j: v_{j} \in[1, m], \quad \sum_{i} h_{i}=\sum_{j} v_{j}$.

For $\min (W), \min (E)=1, \cdots, m$ and 
$\min (N), \min (S)=1, \cdots, n$ do begin

If $\Delta_{2 L, \text { Geo }}^{1,1}(H, V)$ or $\Delta_{2 L, G \text { Geo }}^{1,1}(H, V)$ or...or $\Delta_{2 L, L G e 07,2 L G E O 7}^{1,1}(H, V)$ is satisfiable,

then output $P=\overline{A \cup B \cup C \cup D}$ and halt.

end

output "failure".

\subsection{Clauses for the Class $\chi_{2 L}^{1,1}$}

We code by a 2SAT formula the fourteen geometries that characterize all 2-convex polyominoes in the class $\chi_{2 L}^{1,1}$ in order to reconstruct them.

$$
\begin{aligned}
& \text { Pos }=\left\{C_{(\min (E), 1)} \wedge C_{(m, \max (N))} \wedge A_{1,1} \wedge D_{m, n}\right\}
\end{aligned}
$$

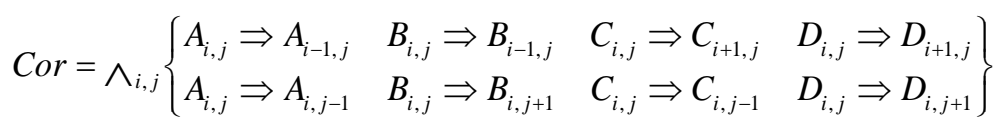

$$
\begin{aligned}
& \text { Dis }=\wedge_{i, j}\left\{X_{i, j} \Rightarrow \bar{Y}_{i, j} \text { : for symbols } X, Y \in\{A, B, C, D\}, X \neq Y\right\} \\
& \text { Con }=\wedge_{i, j}\left\{A_{i, j} \Rightarrow \bar{D}_{i+1, j+1} \quad B_{i, j} \Rightarrow \bar{C}_{i+1, j-1}\right\} \\
& A n C=\left\{\begin{array}{l}
\bar{A}_{\min (W), 1} \wedge \bar{A}_{\min (E), n} \wedge \bar{B}_{\min (W), 1} \wedge \bar{B}_{\min (E), n} \wedge \\
\bar{C}_{\min (W), 1} \wedge \bar{C}_{\min (E), n} \wedge \bar{D}_{\min (W), 1} \wedge \bar{D}_{\min (E), n} \wedge \\
\bar{A}_{1, \min (N)} \wedge \bar{A}_{m, \min (S)} \wedge \bar{B}_{1, \min (N)} \wedge \bar{B}_{m, \min (S)} \wedge \\
\bar{C}_{1, \min (N)} \wedge \bar{C}_{m, \min (S)} \wedge \bar{D}_{1, \min (N)} \wedge \bar{D}_{m, \min (S)} \wedge \\
\bar{A}_{\max (W), 1} \wedge \bar{A}_{\max (E), n} \wedge \bar{B}_{\max (W), 1} \wedge \bar{B}_{\max (E), n} \wedge \\
\bar{C}_{\max (W), 1} \wedge \bar{C}_{\max (E), n} \wedge \bar{D}_{\max (W), 1} \wedge \bar{D}_{\max (E), n} \wedge \\
\bar{A}_{1, \max (N)} \wedge \bar{A}_{m, \max (S)} \wedge \bar{B}_{1, \max (N)} \wedge \bar{B}_{m, \max (S)} \wedge \\
\bar{C}_{1, \max (N)} \wedge \bar{C}_{m, \max (S)} \wedge \bar{D}_{1, \max (N)} \wedge \bar{D}_{m, \max (S)}
\end{array}\right\} \\
& L B C=\wedge_{i}\left\{\begin{array}{cc}
\wedge_{j<\min (N)} A_{i, j} \Rightarrow \bar{C}_{i+v_{j}, j} & \wedge_{\min (S) \leq j \leq \max (S)} B_{i, j} \Rightarrow \bar{C}_{i+v_{j}, j} \\
\wedge_{\min (N) \leq j \leq \max (N)} C_{i+v_{j}, j} \Rightarrow \bar{A}_{i, j} & \wedge_{j>\max (S)} B_{i, j} \Rightarrow \bar{D}_{i+v_{j}, j} \\
\wedge_{\max (N)<j<\min (S)} B_{i, j} \Rightarrow \bar{C}_{i+v_{j}, j} &
\end{array}\right\} \\
& \wedge \wedge_{j}\left\{\bar{C}_{v_{j}, j} \quad \bar{D}_{v_{j}, j}\right\} \\
& U B R=\wedge_{j}\left\{\begin{array}{cc}
\wedge_{i<\min (E)} \bar{A}_{i, j} \Rightarrow B_{i, j+h_{i}} & \wedge_{\min (W) \leq i \leq \max (W)} \bar{A}_{i, j} \Rightarrow D_{i, j+h_{i}} \\
\wedge_{\min (E) \leq i \leq \max (E)} \bar{B}_{i, j+h_{i}} \Rightarrow A_{i, j} & \wedge_{i>\max (W)} \bar{C}_{i, j} \Rightarrow D_{i, j+h_{i}} \\
\wedge_{\max (E)<i<\min (W)} \bar{A}_{i, j} \Rightarrow D_{i, j+h_{i}} &
\end{array}\right\} \\
& R E C=\left\{\bar{A}_{\min (W)-1, \min (N)-1} \wedge \bar{D}_{\max (E)+1, \max (S)+1}\right\} \\
& R E C 1=\left\{\begin{array}{c}
A_{\min (W)-1, \min (N)-2} \wedge A_{\min (W)-2, \min (N)-1} \wedge D_{\max (E)+1, \max (S)+2} \\
\wedge D_{\max (E)+2, \max (S)+1}
\end{array}\right\} \\
& G E O 1=\left\{\begin{array}{c}
\bar{A}_{\max (E), \max (N)} \wedge \bar{B}_{\max (E), \max (N)} \wedge \bar{C}_{\max (E), \max (N)} \\
\wedge \bar{D}_{\max (E), \max (N)} \wedge \bar{A}_{\max (W), \max (S)+1} \wedge \bar{B}_{\max (W), \max (S)+1} \\
\wedge \bar{C}_{\max (W), \max (S)+1} \wedge \bar{D}_{\max (W), \max (S)+1}
\end{array}\right\}
\end{aligned}
$$




$$
\begin{aligned}
& L G E O 2=\left\{\begin{array}{c}
\bar{A}_{\min (E), \min (N)} \wedge \bar{B}_{\min (E), \min (N)} \wedge \bar{C}_{\min (E), \min (N)} \\
\wedge \bar{D}_{\min (E), \min (N)} \wedge \bar{A}_{\min (W), \max (S)+1} \wedge \bar{B}_{\min (W), \max (S)+1} \\
\wedge \bar{C}_{\min (W), \max (S)+1} \wedge \bar{D}_{\min (W), \max (S)+1}
\end{array}\right\} \\
& 2 L G E O 2=\left\{\begin{array}{c}
B_{\min (W)-1, j} \Rightarrow \bar{C}_{\max (E), j-1} \wedge{ }_{j>1} \\
\bar{X}_{\min (W)-1, \max (N)+1} \wedge \bar{X}_{\max (E), \max (N)+1}, \forall X \in\{A, B, C, D\}
\end{array}\right\} \\
& \text { GEO3 }=\left\{\begin{array}{c}
\bar{A}_{\min (E), \min (N)} \wedge \bar{B}_{\min (E), \min (N)} \wedge \bar{C}_{\min (E), \min (N)} \\
\wedge \bar{D}_{\min (E), \min (N)} \wedge \bar{A}_{\min (W)-1, \min (S)} \wedge \bar{B}_{\min (W)-1, \min (S)} \\
\wedge \bar{C}_{\min (W)-1, \min (S)} \wedge \bar{D}_{\min (W)-1, \min (S)}
\end{array}\right\} \\
& \text { LGEO4 }=\left\{\begin{array}{c}
\bar{A}_{\max (E), \max (N)} \wedge \bar{B}_{\max (E), \max (N)} \wedge \bar{C}_{\max (E), \max (N)} \\
\wedge \bar{D}_{\max (E), \max (N)} \wedge \bar{A}_{\min (W)-1, \min (S)} \wedge \bar{B}_{\min (W)-1, \min (S)} \\
\wedge \bar{C}_{\min (W)-1, \min (S)} \wedge \bar{D}_{\min (W)-1, \min (S)}
\end{array}\right\} \\
& \text { 2LGEO4 }=\left\{\begin{array}{c}
C_{i, \min (N)} \Rightarrow \bar{B}_{i-1, \max (S)+1} \wedge \wedge_{i>1} \\
\bar{X}_{\max (W)+1, \min (N)} \wedge \bar{X}_{\min (E)-1, \max (S)+1}, \forall X \in\{A, B, C, D\}
\end{array}\right\} \\
& \text { GEO5 }=\left\{\begin{array}{c}
\bar{A}_{\max (W), \max (S)} \wedge \bar{B}_{\max (W), \max (S)} \wedge \bar{C}_{\max (W), \max (S)} \\
\wedge \bar{D}_{\max (W), \max (S)} \wedge \bar{A}_{\max (E)+1, \max (N)} \wedge \bar{B}_{\max (E)+1, \max (N)} \\
\wedge \bar{C}_{\max (E)+1, \max (N)} \wedge \bar{D}_{\max (E)+1, \max (N)}
\end{array}\right\} \\
& \text { LGEO6 }=\left\{\begin{array}{c}
\bar{A}_{\min (W), \min (S)} \wedge \bar{B}_{\min (W), \min (S)} \wedge \bar{C}_{\min (W), \min (S)} \\
\wedge \bar{D}_{\min (W), \min (S)} \wedge \bar{A}_{\max (E)+1, \max (N)} \wedge \bar{B}_{\max (E)+1, \max (N)} \\
\wedge \bar{C}_{\max (E)+1, \max (N)} \wedge \bar{D}_{\max (E)+1, \max (N)}
\end{array}\right\} \\
& \text { 2LGEO6 }=\left\{\begin{array}{c}
C_{i, \min (N)-1} \Rightarrow \bar{B}_{i-1, \max (S)} \wedge_{i>1} \\
\bar{X}_{\max (W)+1, \min (N)-1} \wedge \bar{X}_{\min (E)-1, \max (S)}, \forall X \in\{A, B, C, D\}
\end{array}\right\} \\
& \text { GEO7 }=\left\{\begin{array}{c}
\bar{A}_{\min (W), \min (S)} \wedge \bar{B}_{\min (W), \min (S)} \wedge \bar{C}_{\min (W), \min (S)} \\
\wedge \bar{D}_{\min (W), \min (S)} \wedge \bar{A}_{\min (W)-1, \min (E)} \wedge \bar{B}_{\min (W)-1, \min (E)} \\
\wedge \bar{C}_{\min (W)-1, \min (E)} \wedge \bar{D}_{\min (W)-1, \min (E)}
\end{array}\right\} \\
& L G E O 8=\left\{\begin{array}{c}
\bar{A}_{\max (W), \max (S)} \wedge \bar{B}_{\max (W), \max (S)} \wedge \bar{C}_{\max (W), \max (S)} \\
\wedge \bar{D}_{\max (W), \max (S)} \wedge \bar{A}_{\min (W)-1, \min (E)} \wedge \bar{B}_{\min (W)-1, \min (E)} \\
\wedge \bar{C}_{\min (W)-1, \min (E)} \wedge \bar{D}_{\min (W)-1, \min (E)}
\end{array}\right\} \\
& 2 L G E O 8=\left\{\begin{array}{c}
B_{\min (W), j} \Rightarrow \bar{C}_{\max (E)+1, j-1} \wedge_{j>1} \\
\bar{X}_{\min (W), \min (N)+1} \wedge \bar{X}_{\max (E)+1, \min (S)-1}, \forall X \in\{A, B, C, D\}
\end{array}\right\} \\
& \text { LGEO9 }=\left\{\begin{array}{c}
\bar{A}_{\min (E), \min (N)} \wedge \bar{B}_{\min (E), \min (N)} \wedge \bar{C}_{\min (E), \min (N)} \\
\bar{D}_{\min (E), \min (N)} \wedge \bar{A}_{\max (W), \max (S)} \wedge \bar{B}_{\max (W), \max (S)} \\
\wedge \bar{C}_{\max (W), \max (S)} \wedge \bar{D}_{\max (W), \max (S)}
\end{array}\right\}
\end{aligned}
$$




$$
\begin{aligned}
& 2 L G E O 9=\left\{\begin{array}{c}
B_{\min (W)-1, j} \Rightarrow \bar{C}_{\max (E)+1, j-1} \wedge_{j>1} \\
\bar{X}_{\min (W)-1, \max (N)+1} \wedge \bar{X}_{\max (E)+1, \min (S)-1}, \forall X \in\{A, B, C, D\}
\end{array}\right\} \\
& L G E O 10=\left\{\begin{array}{c}
\bar{A}_{\max (E), \max (N)} \wedge \bar{B}_{\max (E), \max (N)} \wedge \bar{C}_{\max (E), \max (N)} \\
\wedge \bar{D}_{\max (E), \max (N)} \wedge \bar{A}_{\max (W), \max (S)} \wedge \bar{B}_{\max (W), \max (S)} \\
\wedge \bar{C}_{\max (W), \max (S)} \wedge \bar{D}_{\max (W), \max (S)}
\end{array}\right\} \\
& 2 L_{1} G E O 10=\left\{\begin{array}{c}
B_{\min (W)-1, j} \Rightarrow \bar{C}_{\max (E)+1, j-1} \wedge_{j>1} \\
\bar{X}_{\min (W)-1, \max (N)+1} \wedge \bar{X}_{\max (E)+1, \min (S)-1}, \forall X \in\{A, B, C, D\}
\end{array}\right\} \\
& 2 L_{2} G E O 10=\left\{\begin{array}{c}
C_{i, \min (N)} \Rightarrow \bar{B}_{i-1, \max (S)+1} \wedge_{i>1} \\
\bar{X}_{\max (W)+1, \min (N)} \wedge \bar{X}_{\min (E)-1, \max (S)+1}, \forall X \in\{A, B, C, D\}
\end{array}\right\} \\
& L G E O 11=\left\{\begin{array}{c}
\bar{A}_{\min (W), \min (S)} \wedge \bar{B}_{\min (W), \min (S)} \wedge \bar{C}_{\min (W), \min (S)} \\
\bar{D}_{\min (W), \min (S)} \wedge \bar{A}_{\min (E), \min (N)} \wedge \bar{B}_{\min (E), \min (N)} \\
\wedge \bar{C}_{\min (E), \min (N)} \wedge \bar{D}_{\min (E), \min (N)}
\end{array}\right\} \\
& 2 L_{1} G E O 11=\left\{\begin{array}{c}
B_{\min (W)-1, j} \Rightarrow \bar{C}_{\max (E)+1, j-1} \wedge_{j>1} \\
\bar{X}_{\min (W)-1, \max (N)+1} \wedge \bar{X}_{\max (E)+1, \min (S)-1}, \forall X \in\{A, B, C, D\}
\end{array}\right\} \\
& 2 L_{2} G E O 11=\left\{\begin{array}{c}
C_{i, \min (N)-1} \Rightarrow \bar{B}_{i-1, \max (S)} \wedge_{i>1} \\
\bar{X}_{\max (W)+1, \min (N)-1} \wedge \bar{X}_{\min (E)-1, \max (S)}, \forall X \in\{A, B, C, D\}
\end{array}\right\} \\
& L G E O 12=\left\{\begin{array}{c}
\bar{A}_{\min (W), \min (S)} \wedge \bar{B}_{\min (W), \min (S)} \wedge \bar{C}_{\min (W), \min (S)} \\
\wedge \bar{D}_{\min (W), \min (S)} \wedge \bar{A}_{\max (E), \max (N)} \wedge \bar{B}_{\max (E), \max (N)} \\
\wedge \bar{C}_{\max (E), \max (N)} \wedge \bar{D}_{\max (E), \max (N)}
\end{array}\right\} \\
& 2 L G E O 12=\left\{\begin{array}{c}
C_{i, \min (N)-1} \Rightarrow \bar{B}_{i-1, \max (S)+1} \wedge_{i>1} \\
\bar{X}_{\max (W)+1, \min (N)-1} \wedge \bar{X}_{\max (E)+1, \max (S)+1}, \forall X \in\{A, B, C, D\}
\end{array}\right\} \\
& L G E O 13=\left\{\begin{array}{c}
\bar{A}_{\max (E), \max (N)} \wedge \bar{B}_{\max (E), \max (N)} \wedge \bar{C}_{\max (E), \max (N)} \\
\wedge \bar{D}_{\max (E), \max (N)} \wedge \bar{A}_{\max (W), \max (S)} \wedge \bar{B}_{\max (W), \max (S)} \\
\wedge \bar{C}_{\max (W), \max (S)} \wedge \bar{D}_{\max (W), \max (S)}
\end{array}\right\} \\
& 2 L_{1} G E O 13=\left\{\begin{array}{c}
B_{\min (W), j} \Rightarrow \bar{C}_{\max (E)+1, j-1} \wedge_{j>1} \\
\bar{X}_{\min (W), \max (N)+1} \wedge \bar{X}_{\max (E)+1, \min (S)-1}, \forall X \in\{A, B, C, D\}
\end{array}\right\} \\
& 2 L_{2} G E O 13=\left\{\begin{array}{c}
C_{i, \min (N)-1} \Rightarrow \bar{B}_{i-1, \max (S)+1} \wedge_{i>1} \\
\bar{X}_{\max (W)+1, \min (N)-1} \wedge \bar{X}_{\max (E)+1, \max (S)+1}, \forall X \in\{A, B, C, D\}
\end{array}\right\} \\
& L G E O 14=\left\{\begin{array}{c}
\bar{A}_{\min (E), \min (N)} \wedge \bar{B}_{\min (E), \min (N)} \wedge \bar{C}_{\min (E), \min (N)} \\
\wedge \bar{D}_{\min (E), \min (N)} \wedge \bar{A}_{\min (W), \min (S)} \wedge \bar{B}_{\min (W), \min (S)} \\
\wedge \bar{C}_{\min (W), \min (S)} \wedge \bar{D}_{\min (W), \min (S)}
\end{array}\right\}
\end{aligned}
$$




$$
\begin{aligned}
& 2 L_{1} G E O 14=\left\{\begin{array}{c}
B_{\min (W)-1, j} \Rightarrow \bar{C}_{\max (E)+1, j-1} \wedge_{j>1} \\
\bar{X}_{\min (W)-1, \max (N)+1} \wedge \bar{X}_{\max (E) \min (S)-1}, \forall X \in\{A, B, C, D\}
\end{array}\right\} \\
& 2 L_{2} G E O 14=\left\{\begin{array}{c}
C_{i, \min (N)-1} \Rightarrow \bar{B}_{i-1, \max (S)+1} \wedge_{i>1} \\
\bar{X}_{\max (W)+1, \min (N)-1} \wedge \bar{X}_{\max (E)+1, \max (S)+1}, \forall X \in\{A, B, C, D\}
\end{array}\right\}
\end{aligned}
$$

In order to reconstruct all 2-convex polyominoes in the class $\chi_{2 L}^{1,1}$, we use the set of clauses that impose the 14 geometries.

$$
\begin{aligned}
& \chi_{2 L, G e o 1}^{1,1}(H, V)=P o s \wedge \text { Cor } \wedge \text { Dis } \wedge \text { Con } \wedge A n c \wedge L B C \wedge U B R \\
& \wedge R E C \wedge R E C 1 \wedge G E O 1 . \\
& \chi_{2 L, L G e o 2,2 L G E O 2}^{1,1}(H, V)=P o s \wedge \text { Cor } \wedge \text { Dis } \wedge \text { Con } \wedge \text { Anc } \wedge L B C \wedge U B R \\
& \wedge R E C \wedge R E C 1 \wedge L G E O 2 \wedge 2 L G E O 2 . \\
& \chi_{2 L, \text { Geo3 }}^{1,1}(H, V)=P \text { os } \wedge \text { Cor } \wedge \text { Dis } \wedge \text { Con } \wedge \text { Anc } \wedge L B C \wedge U B R \\
& \wedge R E C \wedge R E C 1 \wedge G E O 3 . \\
& \chi_{2 L, L G e o 4,2 L G E O 4}^{1,1}(H, V)=P o s \wedge \text { Cor } \wedge \text { Dis } \wedge \text { Con } \wedge \text { Anc } \wedge L B C \wedge U B R \\
& \wedge R E C \wedge R E C 1 \wedge L G E O 4 \wedge 2 L G E O 4 . \\
& \chi_{2 L, \text { Geo }}^{1,1}(H, V)=P o s \wedge \text { Cor } \wedge \text { Dis } \wedge \text { Con } \wedge A n c \wedge L B C \wedge U B R \\
& \wedge R E C \wedge R E C 1 \wedge G E O 5 . \\
& \chi_{2 L, L G e o 6,2 L G E O 6}^{1,1}(H, V)=P o s \wedge C o r \wedge \text { Dis } \wedge \text { Con } \wedge A n c \wedge L B C \wedge U B R \\
& \wedge R E C \wedge R E C 1 \wedge L G E O 6 \wedge 2 L G E O 6 . \\
& \chi_{2 L, G e o 7}^{1,1}(H, V)=P \text { os } \wedge \text { Cor } \wedge \text { Dis } \wedge \text { Con } \wedge A n c \wedge L B C \wedge U B R \\
& \wedge R E C \wedge R E C 1 \wedge G E O 7 . \\
& \chi_{2 L, L G e o 8,2 L G E O 8}^{1,1}(H, V)=P o s \wedge C o r \wedge \text { Dis } \wedge \text { Con } \wedge \text { Anc } \wedge L B C \wedge U B R \\
& \wedge R E C \wedge R E C 1 \wedge L G E O 8 \wedge 2 L G E O 8 . \\
& \chi_{2 L, L G e o 9,2 L G E O 9}^{1,1}(H, V)=P o s \wedge \text { Cor } \wedge \text { Dis } \wedge \text { Con } \wedge A n c \wedge L B C \wedge U B R \\
& \wedge R E C \wedge R E C 1 \wedge L G E O 9 \wedge 2 L G E O 9 . \\
& \chi_{2 L, L G e o 10,2 L_{1} G E O 10,2 L_{2} G E O 10}^{1,1}(H, V) \\
& =P \text { os } \wedge \text { Cor } \wedge \text { Dis } \wedge \text { Con } \wedge \text { Anc } \wedge L B C \wedge U B R \wedge R E C \\
& \wedge R E C 1 \wedge L G E O 10 \wedge 2 L_{1} G E O 10 \wedge 2 L_{2} G E O 10 . \\
& \chi_{2 L, L G e 011,2 L_{1} G E O 11,2 L_{2} G E O 11}^{1,1}(H, V) \\
& =P \text { os } \wedge \text { Cor } \wedge \text { Dis } \wedge \text { Con } \wedge A n c \wedge L B C \wedge U B R \wedge R E C \\
& \wedge R E C 1 \wedge L G E O 11 \wedge 2 L_{1} G E O 11 \wedge 2 L_{2} G E O 11 \text {. } \\
& \chi_{2 L, L G e 012,2 L G E O 12}^{1,1}(H, V)=P o s \wedge C o r \wedge D i s \wedge C o n \wedge A n c \wedge L B C \wedge U B R \\
& \wedge R E C \wedge R E C 1 \wedge L G E O 12 \wedge 2 L G E O 12 . \\
& \chi_{2 L, L G e o 13,2 L_{1} G E O 13,2 L_{2} G E O 13}^{1,1}(H, V) \\
& =P o s \wedge C o r \wedge D i s \wedge C o n \wedge A n c \wedge L B C \wedge U B R \wedge R E C \\
& \wedge R E C 1 \wedge L G E O 13 \wedge 2 L_{1} G E O 13 \wedge 2 L_{2} G E O 13 \text {. }
\end{aligned}
$$




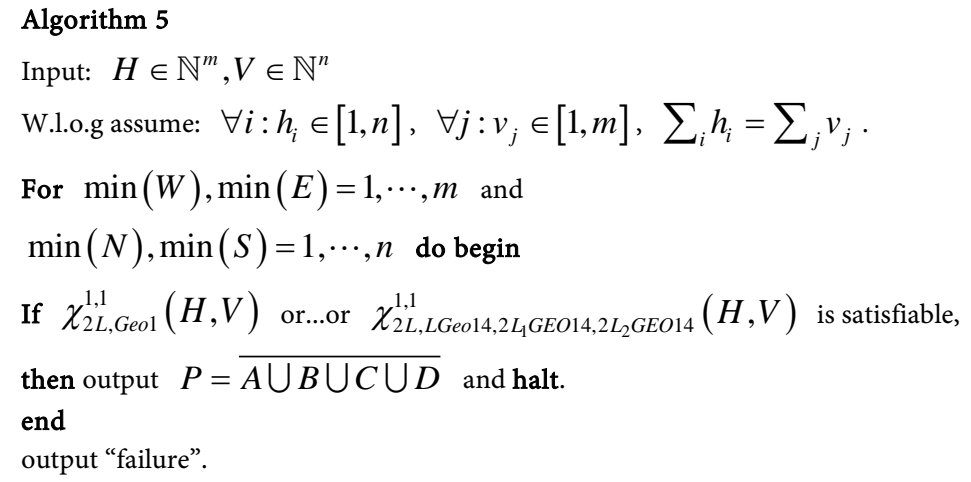

\subsection{Reconstruction of the Class $\beta_{2 L}^{1,1}$ Using the Horizontal Reflexion $S_{H}$}

Given two integer vectors $H=\left(h_{1}, \cdots, h_{m}\right)$ and $V=\left(v_{1}, \cdots, v_{n}\right)$. To reconstruct a polyomino $P$ in the class $\beta_{2 L}^{1,1}$, one can see that the horizontal reflexion $S_{H}:(i, j) \rightarrow(m-i+1, j), \forall i, j \in\{1, \cdots, m\} \times\{1, \cdots, n\}$ sends the projection vectors $(H, V)$ to $(\tilde{H}, V)$, where $\tilde{H}=\left(h_{m}, \cdots, h_{1}\right)$. Now from the two vectors of projections $(\tilde{H}, V)$, one can reconstruct the polyomino $P$ in the class $\alpha_{2 L}^{1,1}$ and then by the horizontal reflexion $S_{H}$, we reconstruct $P$ in the class $\beta_{2 L}^{1,1}$.

\begin{tabular}{ccc}
\hline N,S & $W, E$ \\
$S \rightarrow N$ & $W \rightarrow W$ & $E \rightarrow E$ \\
$N \rightarrow S$ & $\leq \Leftrightarrow \geq$ & $\min \rightarrow \max$ \\
& $\max \rightarrow \min$ \\
\hline
\end{tabular}

\section{Conflicts of Interest}

The authors declare no conflicts of interest regarding the publication of this paper.

\section{References}

[1] Barcucci, E., Del Lungo, A., Nivat, M. and Pinzani, R. (1996) Reconstructing Convex Polyominoes from Horizontal and Vertical Projections. Theoretical Computer Science, 155, 321-347. https://doi.org/10.1016/0304-3975(94)00293-2

[2] Brunetti, S. and Daurat, A. (2005) Random Generation of Q-Convex Sets. Theoretical Computer Science, 347, 393-414. https://doi.org/10.1016/j.tcs.2005.06.033

[3] Castiglione, G., Restivo, A. and Vaglica, R. (2006) A Reconstruction Algorithm for L-Convex Polyominoes. Theoretical Computer Science, 356, 58-72. 
http://www.sciencedirect.com https://doi.org/10.1016/j.tcs.2006.01.045

[4] Castiglione, G., Frosini, A., Munarini, E., Restivo, A. and Rinaldi, S. (2007) Combinatorial Aspects of $L$-Convex Polyominoes. European Journal of Combinatorics, 28, 1724-1741.

[5] Castiglione, G. and Restivo, A. (2003) Reconstruction of L-Convex Polyominoes. Electronic Notes in Discrete Mathematics, Vol. 12, Elsevier Science, Amsterdam. https://doi.org/10.1016/S1571-0653(04)00494-9

[6] Duchi, E., Rinaldi, S. and Schaeffer, G. (2008) The Number of Z-Convex Polyominoes. Advances in Applied Mathematics, 40, 54-72. https://doi.org/10.1016/j.aam.2006.07.004

[7] Tawbe, K. and Vuillon, L. (2011) 2L-Convex Polyominoes: Geometrical Aspects. Contributions to Discrete Mathematics, North America, 6, 1-25.

[8] Chrobak, M. and Dürr, C. (1999) Reconstructing hv-Convex Polyominoes from Orthogonal Projections. Information Processing Letters, 69, 283-289.

https://doi.org/10.1016/S0020-0190(99)00025-3

[9] Tawbe, K., Ghandour, N. and Atwi, A. (2019) 2-Convex Polyominoes: Non-Empty Corners. Open Journal of Discrete Mathematics, 9, 33-51.

[10] Tawbe, K. and Vuillon, L. (2011) 2 L-Convex Polyominoes: Tomographical Aspects. Contributions to Discrete Mathematics, 8, 1-12.

[11] Even, S., Itai, A. and Shamir, A. (1976) On the Complexity of Timetable and Multicommodity Flow Problems. SIAM Journal on Computing, 5, 691-703. https://doi.org/10.1137/0205048

[12] Aspvall, B., Plass, M.F. and Tarjan, R.E. (1979) A Linear-Time Algorithm for Testing the Truth of Certain Quantified Boolean Formulas. Information Processing Letters, 8, 121-123. https://doi.org/10.1016/0020-0190(79)90002-4 